\title{
m-ISOMETRIC TRANSFORMATIONS OF HILBERT SPACE, I
}

Jim Agler and Mark Stankus

Department of Mathematics, Univ. Of Calif., San Diego

La Jolla, CA 92093-0112, U.S.A.

A model for operators satisfying the equation $\sum_{k=0}^{m}(-1)^{k}\left(\begin{array}{c}m \\ k\end{array}\right)\left(T^{*}\right)^{m-k} T^{m-k}=0$ is given as multiplication by $e^{i \phi}$ on a Hilbert space whose inner product is defined in terms of periodic distributions and we relate this model theory for the case when $m=2$ to a disconjugacy theory for a subclass of Toeplitz operators of the type studied by Boutet de Monvel and Guilliman, classical function theoretic ideas on the Dirichlet space, and the theory of nonstationary stochastic processes. This is presented in a series of three papers. In this first paper, we concentrate on a model for these $T$.

\section{Table of Contents}

\section{Paper I.}

0. Introduction

1. The Elementary Operator Theory of $m$-Isometries

2. Distribution Differential Operators on the circle

3. A model for $m$-isometries

4. Smoothing applied to $m$-isometries

\section{Paper II.}

Introduction to Paper II

5. Brownian shifts, Brownian unitaries and Brownian isometries

6. A Disconjugacy Theorem for Matricial Toeplitz Operators

7. Another proof of the Lifting Theorem

\section{Paper III.}

Introduction to Paper III

8. A cyclic vector

9. Brownian Unitaries Revisited

10. Some remarks and Open Questions 


\section{$\S 0$. Introduction}

In this paper we shall study the bounded linear transformations $T$ of a complex Hilbert space $\mathcal{H}$ that satisfy an identity of the form

$$
T^{* m} T^{m}-\left(\begin{array}{c}
m \\
1
\end{array}\right) T^{*^{m-1}} T^{m-1}+\left(\begin{array}{c}
m \\
2
\end{array}\right) T^{*^{m-2}} T^{m-2}-\cdots+(-1)^{m}=0
$$

for some positive integer $m$. Operators $T$ satisfying (0.1) are said to be $m$-isometries. The case when $m=1$ has already received definitive analysis by operator theorists: a circumstance due in large part to the fundamental importance of an adequate theory of isometries to the intrinsic problem of modeling the general contractive operator via its isometric dilation ([Br-R], [Sz.-N-F] $)$ as well as to the development of a successful $L^{2}$ theory for stationary stochastic processes, to the $H^{\infty}$-control problem [H3], and to the function theory of the classical Hardy space [B,Hel]. To develop an analysis of $m$ isometries in the case when $m>1$ requires ideas from many areas of mathematics. In particular, in this paper we shall employ the theory of periodic distributions to derive a function theory model for $m$-isometries, a disconjugacy theory for a subclass of Toeplitz operators of the type studied by Boutet de Monvel and Guillemin [M-G] and function theoretic ideas on the classical Dirichlet space. In addition, operators arising from a certain class of nonstationary stochastic processes related to Brownian motion will play an essential role in the theory of 2-isometries.

Our basic approach will be based on two ideas of J. W. Helton who in [H1] initiated the study of operators $T$ which satisfy an identity of the form

$$
T^{* m}-\left(\begin{array}{c}
m \\
1
\end{array}\right) T^{*^{m-1}} T+\cdots+(-1)^{m} T^{m}=0 .
$$

Helton's first idea was that such operators possess a model as multiplication operators on Hilbert spaces of $C^{\infty}$-functions completed with respect to a inner product

$$
[\varphi, \psi]=\int L(\varphi) \bar{\psi}
$$

where $L$ is a positive definite differential operator on a compact interval $I \subseteq \mathbb{R}$. The second idea [H2] was that the general operator $T$ satisfying $(0.2)$ can be explicitly represented in the form

$$
T=J \mid \mathcal{M}
$$

where $J$ is a real jordan operator (i.e., $J=A+Q, A=A^{*}, Q A=A Q$, and $Q$ is nilpotent) and that such a representation is formally equivalent to the classical disconjugacy theory for elliptic ODE. In this paper we shall carry out Helton's first idea for the case of $m$-isometries. This results in a general model for the finitely cyclic $m$-isometry as multiplication by $e^{i \theta}$ on the completion of a pre-Hilbert space of analytic functions with respect to an inner 
product as in (0.3) where $L$ is a differential operator on $\partial \mathrm{D}$ whose compression to the analytic $C^{\infty}$-functions is positive definite. In addition we shall carry out Helton's second idea for the case $m=2$. This results in a theorem that represents the general 2-isometry $T$ in the form

$$
T=B \mid \mathcal{M}
$$

where $B$ has the block form

$$
B=\left[\begin{array}{cc}
V & \sigma E \\
0 & U
\end{array}\right],
$$

$\sigma>0$ is a constant, $V$ is an isometry, $U$ is unitary and $E$ is a Hilbert space isomorphism onto ker $V^{*}$. The operators $B$ of the form in (0.4) are referred to as Brownian unitaries of covariance $\sigma$ and were discovered by considering certain natural examples of 2-isometries arising from statistics.

The paper is divided into ten sections and is published in three parts. The first part consists of Sections 1-4 and deals with the general theory of $m$-isometries, the second part consists of Sections 5-7 and prove a spatial lifting theorem for 2-isometries. and the third part consists of Sections 8 -10. Sections 8 and 9 analyze cyclic 2 -isometries in more detail and state a number of open questions related to $m$-isometries. Although the paper is quite long the reader should not conclude that the theory of $m$-isometries (even in the case when $m=2$ ) is any way complete. In Section 10 we discuss a number of unsolved problems and possible directions for further research.

In Section 1 we discuss the elementary operator theory of $m$-isometries. It turns out that if $T$ is an $m$-isometry and $\Delta_{T}$ is defined by

$$
\Delta_{T}=\left(T^{*}\right)^{m-1} \cdot T^{m-1}-\left(\begin{array}{c}
m-1 \\
1
\end{array}\right)\left(T^{*}\right)^{m-2} T^{m-2}+\ldots+(-1)^{m-1}
$$

then $\Delta_{T}$ is a positive operator, kernel $\Delta_{T}$ invariant for $\mathrm{T}$ and $T \mid \operatorname{ker} \Delta_{T}$ is an $m-1$ - isometry. These facts can be milked for a considerable amount of information. In particular, several notions of purity are introduced and several canonical block matrix representations for $m$-isometries are presented. In addition, if $m=2$ and $T$ is finitely cyclic, then $\Delta_{T}$ is compact (c.f. [P] [Ber-S]).

The spectral picture of an $m$-isometry is easy to describe. If $T$ is an $m$-isometry, then $\sigma_{a p}(T) \subseteq \partial \mathbb{D}$ and so $\sigma(T) \subseteq \partial \mathbb{D}$ or $\sigma(T)=\mathbb{D}^{-}$(Lemma 1.21). Consequently, $\sigma_{l e}(T) \subseteq \partial \mathbb{D}$ and $T-\lambda$ is semi-Fredholm for each $\lambda \in \mathbb{D}$ and in particular,

$$
\operatorname{ind}(T-\lambda) \equiv \operatorname{ind} T \leq 0
$$

for all $\lambda \in \mathbb{D}$.

In particular there is the following spectral dichotomy: if $T$ is invertible, then $\sigma(T) \subseteq$ $\partial \mathrm{D}$; if $T$ is not invertible, then $\sigma(T)=\mathbb{D}^{-}$. In both cases it turns out that there is a 
functional calculus (Proposition 1.20). In the invertible case the functional calculus is a continuous homomorphism of $\mathcal{D}$, the algebra of $C^{\infty}$ functions defined on the unit circle, and in the noninvertible case the calculus is a continuous homomorphism on $\mathcal{D}_{a}$, the algebra of analytic functions on the dise with $C^{\infty}$ boundary values.

In Section 2 we introduce the class of distribution differential operators (DDO). A DDO $L$ is a linear mapping from $\mathcal{D}$ into $\mathcal{D}^{\prime}$, the space of periodic distributions, that has the particular form

$$
L(\varphi)=\varphi \beta_{0}+\varphi^{\prime} \beta_{1}+\varphi^{\prime \prime} \beta_{2}+\ldots+\varphi^{(m)} \beta_{m} \quad, \quad \varphi \in \mathcal{D}
$$

for some $\beta_{0}, \ldots, \beta_{m} \in \mathcal{D}^{\prime}$. In (0.5), we follow the usual convention for defining the product of an element in $\mathcal{D}$ by an element in $\mathcal{D}^{\prime}$ by defining $\varphi^{(l)} \beta_{l} \in \mathcal{D}^{\prime}$ by $\varphi^{(l)} \beta_{l}(\psi)=\beta_{l}\left(\varphi^{(l)} \psi\right)$. Thus, a DDO is simply a differential operator on the circle with distribution coefficients and if in (0.5) the coefficients $\beta_{l}$ are functions, then $L$ is a classical differential operator.

The significance of DDO to the theory of $m$-isometries is revealed in Section 3. It turns out that if $T$ is a $m$-isometry with cyclic vector $\gamma$, then there exists a unique DDO $L$ with the property that

$$
\langle\varphi(T) \gamma, \psi(T) \gamma\rangle=L(\varphi)(\bar{\psi})
$$

for all $\varphi, \psi \in \mathcal{D}_{a}$. In (0.6) $\varphi(T)$ and $\psi(T)$ are formed using the functional calculus. (0.6) then allows one to construct a model for the general bicyclic invertible $m$-isometry as multiplication by $e^{i \theta}$ on the form domain of a DDO (Theorem 3.14), and in the noninvertible case to construct a model for the general cyclic $m$-isometry as multiplication by $e^{i \theta}$ on the analytic form domain of a DDO (Theorem 3.23). In the noncyclic cases models are obtainable by considering DDO with matrix coefficients (Theorems 3.44 and 3.49). Finally, the problem of how the DDO $L$ in (0.6) transforms under changes of cyclic vector $\gamma$ is considered in Theorem 3.52 .

Translation and convolution can be extended in a canonical way to DDO and these operations on the DDO $L$ that represent $m$-isometries $T$ via $(0.6)$ correspond to interesting Hilbert space constructions on the operator $T$. In particular through the use of an approximate identity the $L$ attached to a general $m$-isometry via (0.6) can be smoothed and some questions about $m$-isometries reduced to the case when $L$ is a classical differential operator. This technique was first used in [Ag1] to settle the lifting conjecture of Helton concerning 3-symmetric operators (equation (0.2) with $m=3$ ) and in the present context is applied in Section 7 to establish the equivalence of a disconjugacy theory for classical analytic DDO of order 1 (Lemmas 6.7 and 6.16 and Proposition 6.17) and an algebraic lifting theorem for 2-isometries (Theorem 5.80).

Papers II and III of this series are devoted to the study of 2 -isometries. In Section 5 a certain class of 2 -isometries motivated by statistical considerations is introduced, the 
Brownian unitaries. The irreducible Brownian unitaries correspond to the time shift operator on a scaled Brownian motion process and are referred to as Brownian shifts (Definition 5.5). Each Brownian shift is determined by a nonrandom rotation angle $\theta$ and a positive covariance scalar $\sigma$. It turns out that there is a direct integral theory for Brownian unitaries (Theorem 5.20), each is a direct integral with multiplicity over angles of Brownian shifts of a fixed covariance $\sigma$. The scalar $\sigma$ is referred to as the covariance of the Brownian unitary. Furthermore, the Brownian shifts in addition to their appearance as the time shift for perhaps the most ubiquitous of nonstationary Gaussian stochastic processes have elegant characterizations on the level of operator theory (Proposition 5.6) as well as within the field of DDO (Proposition 5.2). The principal result of Section 5, the Lifting Theorem for 2-Isometries (Theorem 5.80), asserts that the general 2-isometry $T$ with $\left\|\Delta_{T}\right\| \leq \sigma^{2}$ can be represented as the restriction to an invariant subspace of a Brownian unitary of covariance $\sigma$.

The proof of the Lifting Theorem in Section 5 consists of a synthesis of a proof of the Sz.-Nagy Dilation Theorem and a proof of the familiar fact that isometries possess extensions to unitaries, and in particular, is purely algebraic in character. The original proof of the Lifting Theorem was analytic in character and derives from first principles the class of objects the 2-isometries are to be lifted to (i.e., the Brownian unitaries). The main analytic content here is a discongugacy theory for regular first order DDO with $L^{\infty}$ coefficients discussed in Section 6 (Lemmas 6.7 and 6.16 and Proposition 6.17).

A second class of operators discussed at length in Section 5 is the collection of direct integrals with multiplicity in $\theta$ of Brownian shifts with varying covariance which we refer to as Brownian isometries. This class not only arises naturally in Theorem 6.20 which studies the extensions of 2-isometries defined as multiplication by $e^{i \theta}$ on the form domain of Toeplitz operators (i.e., (0.5) with $m=1, \beta_{1}=\frac{d \theta}{2 \pi}$, and $\beta_{0}=w \frac{d \theta}{2 \pi}$ with $w \in L^{\infty}$ ) but also arises naturally in the general theory of Brownian unitary extensions which is studied in Section 9. Both the classes of Brownian unitaries and Brownian isometries possess elegant $C^{*}$-algebraic characterizations (Theorems 5.20 and 5.48).

Sections 8 and 9 are devoted to the study of cyclic 2 -isometries. It turns out that if $T$ is a cyclic 2 -isometry, then

$$
\operatorname{dim} \operatorname{ker}\left(\left\|\Delta_{T}\right\|-\Delta_{T}\right)=1
$$

and furthermore if $f_{0} \neq 0$ is chosen so that $\Delta_{T} f_{0}=\left\|\Delta_{T}\right\| f_{0}$, then $f_{0}$ is cyclic for $T$ if and only if $T$ is pure (Theorem 8.19). Here, a 2-isometry $T$ is said to be pure if it has no isometric direct summand. If one chooses $\gamma=f_{0}$, then the DDO $L$ attached to $T$ via (0.6) turns out to have a particularly nice form. This allows one to construct a model for $T$ based on a probability measure on the unit circle (Theorem 8.32). Finally, Section 9 uses Theorem 8.19 to derive a number of qualitative facts about the existence and uniqueness of Brownian unitary and isometric extensions of a cyclic 2-isometry. 
The class of 2-isometries has also been independently studied by Stefan Richter [R1], [R2]. His work develops a Dirichlet space type model for 2-isometries by exploiting a beautiful extension of the wandering subspace argument for isometries to the class of 2isometries type which has considerable overlap with Section 8 of this paper.

This model leads to a rich function theoretic analysis of 2-isometries.

\section{§1. The Elementary Operator Theory of $m$-Isometries}

In this section, we shall introduce much of the notation of the paper and give some basic properties of $m$-isometries. We shall then specialize to the case $m=2$ and obtain additional information.

All Hilbert spaces shall be complex and separable. If $\mathcal{H}$ is a Hilbert space we let $\mathcal{L}(\mathcal{H})$ denote the bounded linear transformations of $\mathcal{H}$. If $T \in \mathcal{L}(\mathcal{H})$ and $m$ is a positive integer we define $(y x-1)^{m}(T) \in \mathcal{L}(\mathcal{H})$ by the formula,

$$
(y x-1)^{m}(T)=\sum_{k=0}^{m}(-1)^{m-k}\left(\begin{array}{c}
m \\
k
\end{array}\right) T^{* k} T^{k} .
$$

If $T \in \mathcal{L}(\mathcal{H})$ and $(y x-1)^{n}(T)=0$, then we shall say that $T$ is an $m$-isometry. A basic fact in the theory of $m$-isometries is that if $T \in \mathcal{L}(\mathcal{H})$ is an $m$-isometry and $\mathcal{M} \subseteq \mathcal{H}$ is an invariant subspace for $T$, then $T \mid \mathcal{M}$ is an $m$-isometry. This follows from the algebraic formula,

$$
(y x-1)^{\ell}(T \mid \mathcal{M})=P_{\mathcal{M}}(y x-1)^{\ell}(T) \mid \mathcal{M}
$$

where $T$ is an arbitrary operator on $\mathcal{H}, \mathcal{M} \subseteq \mathcal{H}$ is invariant for $T, P_{\mathcal{M}}$ denotes the orthogonal projection of $\mathcal{H}$ onto $\mathcal{M}$, and $\ell$ is a nonnegative integer.

A key object in the theory of $m$-isometries is the symbol of $T, s_{T}$, defined for an arbitrary $T \in \mathcal{L}(\mathcal{H})$ by the equation

$$
s_{T}(k)=T^{* k} T^{k} \quad, \quad k \geq 0
$$

and for an invertible $T \in \mathcal{L}(\mathcal{H})$ by the equation

$$
s_{T}(k)=T^{* k} T^{k} \quad, \quad k \in \mathbb{Z} .
$$

Computations involving $s_{T}(k)$ come out quite clearly if we consider the formal difference series for $s_{T}(k)$,

$$
s_{T}(k)=\sum_{\ell=0}^{\infty} \beta_{\ell}(T) k^{(\ell)}
$$


where $k^{(\ell)}=k \cdot(k-1) \cdots(k-\ell+1)$ and

$$
\beta_{\ell}(T)=\frac{1}{\ell !}(y x-1)^{\ell}(T)
$$

If $a=\left\{a_{k}\right\}$ is a sequence of scalars or operators we define a sequence $\delta a$ by setting $\delta a=\left\{a_{k+1}-a_{k}\right\}$. With this notation $\delta\left\{k^{(\ell)}\right\}=(k \geq 0)$, and formula (1.3) is derived by applying $\delta^{\ell}$ to (1.2) and setting $k=0$.

Now observe that if $T \in \mathcal{L}(\mathcal{H})$ and $\beta_{\ell}(T)=0$ then $\beta_{j}(T)=0$ whenever $j \geq \ell$. Hence if $T \in \mathcal{L}(\mathcal{H})$ one sees via (1.3) that $T$ is an $m$-isometry if and only if $s_{T}(k)$ is a polynomial in $k$ of degree less than $m$. A deeper fact that also follows from (1.3) concerns the object of the following definition.

Definition 1.4. If $T \in \mathcal{L}(\mathcal{H})$ is an $m$-isometry, then $\Delta_{T}$, the covariance operator of $T$, is defined by

$$
\triangle_{T}=\beta_{m-1}(T)
$$

Note in the definition of $\Delta_{T}$ we have in our notation suppressed the dependence of $\Delta_{T}$ on $m$.

The following propositions illustrate the interest of $\triangle_{T}$.

Proposition 1.5. If $T$ is an $m$-isometry, then $\triangle_{T} \geq 0$.

Proof. The proof consists of the following computation.

$$
\begin{aligned}
\left\langle\triangle_{T} x, x\right\rangle & =\lim _{k \rightarrow \infty} \frac{1}{k^{(m-1)}}\left\langle s_{T}(k) x, x\right\rangle \\
& =\lim _{k \rightarrow \infty} \frac{1}{k^{(m-1)}}\left\|T^{k} x\right\|^{2} \\
& \geq 0 .
\end{aligned}
$$

Proposition 1.6. If $T \in \mathcal{L}(\mathcal{H})$ is an $m$-isometry, then $\operatorname{ker}\left(\triangle_{T}\right)$ is invariant for $T$ and $T\}$ ker $\triangle_{T}$ is an $m-1$ isometry. Furthermore, if $\mathcal{M} \subseteq \mathcal{H}$ is invariant for $T$ and $T \mid \mathcal{M}$ is an $(m-1)$-isometry, then $\mathcal{M} \subseteq$ ker $\triangle_{T}$.

Proof. Since $T$ is an $m$-isometry,

$$
T^{*} \triangle_{T} T-\triangle_{T}=\beta_{m}(T)=0
$$

and in addition Proposition 1.5 implies that

$$
\Delta_{T} \geq 0
$$


To see that $\operatorname{ker}\left(\triangle_{T}\right)$ is invariant for $T$ observe that if $x \in$ ker $\triangle_{T}$, then

$$
\begin{aligned}
\left\langle\triangle_{T} T x, T x\right\rangle & =\left\langle T^{*} \triangle_{T} T x, x\right\rangle \\
& =\left\langle\triangle_{T} x, x\right\rangle \\
& =0 .
\end{aligned}
$$

That in fact $T \mid$ ker $\triangle_{T}$ is an $(m-1)$-isometry follows from (1.1). Finally, to see that $\mathcal{M} \subseteq$ ker $\triangle_{T}$ whenever $\mathcal{M} \subseteq \mathcal{H}, T \mathcal{M} \subseteq \mathcal{M}$ and $T \mid \mathcal{M}$ is an $(m-1)$-isometry fix $x \in \mathcal{M}$. Using (1.1) we see that

$$
\begin{aligned}
\left\langle\triangle_{\Upsilon} x, x\right\rangle & =\left\langle\beta_{m-1}(T) x, x\right\rangle \\
& =\left\langle\beta_{m-1}(T \mid \mathcal{M}) x, x\right\rangle \\
& =0 .
\end{aligned}
$$

Hence via Proposition 1.5 we conclude that $x \in$ ker $\Delta_{T}$. This completes the proof of Proposition 1.6.

Note that an application of Zorn's Lemma gives that any operator possesses a maximal invariant subspace $\mathcal{M}$ (possibly equal to $\{0\}$ ) such that $T \mid \mathcal{M}$ is an $m$-1-isometry. Proposition 1.6 guarantees that if $T$ is an $m$-isometry, then such an $\mathcal{M}$ is unique (in fact, equal to ker $\Delta_{T}$ ).

In the next proposition we examine maximal reducing subspaces on which a given $m$-isometry is $m-1$-isometric.

Proposition 1.7. If $T \in \mathcal{L}(\mathcal{H})$ is an m-isometry, then there exists a unique subspace $\mathcal{M} \subseteq \mathcal{H}$ that is maximal with respect to the following properties:

(i) $\mathcal{M}$ is reducing for $T$, and

(ii) $T \mid \mathcal{M}$ is an $(m-1)$-isometry.

Proof. The existence of subspaces $\mathcal{M}$ that are maximal with respect to (i) and (ii) follows immediately from Zorn's Lemma. To prove uniqueness it suffices to establish that if $\mathcal{M}_{1}$ and $\mathcal{M}_{2}$ satisfy (i) and (ii), then so also $\mathcal{M}=\left(\mathcal{M}_{1}+\mathcal{M}_{2}\right)^{-}$satisfies (i) and (ii). That $\mathcal{M}$ satisfies (i) is immediate. To see that $\mathcal{M}$ satisfies (ii) first observe that since $T \mid \mathcal{M}_{1}$ and $T \mid \mathcal{M}_{2}$ are $(m-1)$-isometries Proposition 1.6 implies that

$$
\mathcal{M}_{1} \subseteq \text { ker } \triangle_{T} \text { and } \mathcal{M}_{2} \subseteq \text { ker } \triangle_{T}
$$

Hence by another application of Proposition 1.6,

$$
\mathcal{M}=\left(\mathcal{M}_{1}+\mathcal{M}_{2}\right)^{-} \subseteq \operatorname{ker} \triangle_{T}
$$

and (ii) holds for $\mathcal{M}$. This concludes the proof of Proposition 1.7.

Proposition 1.7 motivates the following definition. 
Definition 1.8. Let $T \in \mathcal{L}(\mathcal{H})$ be an $m$-isometry and $\ell$ a positive integer. $T$ is $\ell$-pure if $T$ has no nonzero direct summand which is an $\ell$-isometry. $T$ is pure (as an $m$-isometry) if $T$ is $(m-1)$-pure.

We remark before continuing that if $T$ is an $m$-isometry and not an $(m-1)$-isometry, then $T$ may or may not be pure as an $m$-isometry but is never pure as an $(m+1)$-isometry. Also note that if $T$ is pure as an $m$-isometry, then $T$ is not an $(m-1)$-isometry and in addition, $T$ is $\ell$-pure for all $\ell \leq m-1$. Finally, note that the absence of 0 -isometries on spaces of positive dimension implies that all 1 -isometries are pure, conflicting with the usual use of the term pure in the context of isometries.

We now use Proposition 1.7 to derive a canonical decomposition of an $m$-isometry into a direct sum of pure $\ell$-isometries, $\ell \leq m$. Fix an $m$-isometry $T \in \mathcal{L}(\mathcal{H})$. By Proposition 1.7, there exists a decomposition $\mathcal{H}=\left(\mathcal{H} \ominus \mathcal{H}_{m}\right) \oplus \mathcal{H}_{m}$ with the property that if $\mathcal{M} \subseteq \mathcal{H}$ is reducing for $T$ and $T \mid \mathcal{M}$ is an $(m-1)$-isometry, then $\mathcal{M} \subseteq \mathcal{H} \ominus \mathcal{H}_{m}$. In particular,

$$
\mathcal{H}_{m} \text { is reducing for } T \text {, }
$$

$$
T \mid \mathcal{H}_{m} \text { is a pure } m \text {-isometry (if } \quad \mathcal{H}_{m} \neq\{0\} \text { ) }
$$

and

$$
T \mid \mathcal{H} \ominus \mathcal{H}_{m} \text { is an }(m-1)-\text { isometry }
$$

Now repeat the application of Proposition 1.7 with $T$ replaced by $T \mid \mathcal{H} \ominus \mathcal{H}_{m}$ to obtain a space $\mathcal{H}_{m-1} \subseteq \mathcal{H} \ominus \mathcal{H}_{m}$ such that

$$
\begin{gathered}
\mathcal{H}_{m-1} \text { is reducing for } T \\
T \mid \mathcal{H}_{m-1} \quad \text { is a pure }(m-1) \text { - isometry } \quad\left(\text { if } \quad \mathcal{H}_{m-1} \neq\{0\}\right)
\end{gathered}
$$

and

$$
T \mid \mathcal{H} \ominus\left(\mathcal{H}_{m-1} \oplus \mathcal{H}_{m}\right) \text { is an }(m-2) \text { - isometry }
$$

Continuing in this way we obtain a decomposition,

$$
\mathcal{H}=\mathcal{H}_{1} \oplus \mathcal{H}_{2} \oplus \cdots \oplus \mathcal{H}_{m}
$$

with the properties that

$$
\mathcal{H}_{\ell} \text { is reducing for } T, 1 \leq \ell \leq m \text {, }
$$


and

$$
T \mid \mathcal{H}_{\ell} \quad \text { is a pure } \quad \ell \text {-isometry, } \quad 1 \leq \ell \leq m .
$$

Furthermore, it is easy to see that by the construction that any decomposition satisfying (1.15), (1.16), and (1.17) is unique. In particular, one sees that if $T$ is an $m$-isometry and $\ell \leq m-1$, then there exists a unique maximal reducing subspace $\mathcal{M} \subseteq \mathcal{H}$ such that $T \mid \mathcal{M}$ is an $\ell$-isometry $\left(\mathcal{M}=\mathcal{H}_{1} \oplus \cdots \oplus \mathcal{H}_{\ell}\right)$.

On a pragmatic level the decomposition of $(1.15)$ is of value in that most operator theoretic questions about $m$-isometries will reduce down to questions about pure $\ell$-isometries for $\ell \leq m$.

A construction similar to that just implemented for reducing subspaces using Proposition 1.17 can be carried out for invariant subspaces using Proposition 1.6. This results in a decomposition,

$$
\mathcal{H}=\mathcal{H}_{1} \oplus \cdots \oplus \mathcal{H}_{m}
$$

where $\mathcal{M}_{\ell}=\mathcal{H}_{1} \oplus \cdots \oplus \mathcal{H}_{\ell}$ is invariant for $T, T \mid \mathcal{M}_{\ell}$ is an $\ell$-isometry and $\mathcal{M}_{\ell}$ is maximal with respect to these properties.

If $T$ is an $m$-isometry it turns out that $T$ possesses a $C^{\infty}$-functional calculus. Let $\mathcal{D}$ denote the Frechet algebra of $C^{\infty}$ functions on the circle and define $\mathcal{D}_{a} \subset \mathcal{D}$ by setting

$$
\mathcal{D}_{a}=\{\varphi \in \mathcal{D}: \hat{\varphi}(k)=0 \text { whenever } \quad k<0\} .
$$

We say that $T \in \mathcal{L}(\mathcal{H})$ has a $\mathcal{D}_{a}$-functional calculus if there exists a continuous unital algebra homomorphism $\pi: \mathcal{D}_{a} \rightarrow \mathcal{L}(\mathcal{H})$ such that $\pi(1)=1$ and $\pi\left(e^{i \theta}\right)=T$. If, in addition, $T$ is invertible we say $T$ has a $\mathcal{D}$-functional calculus if there exists a continuous unital algebra homomorphism $\pi: \mathcal{D} \rightarrow \mathcal{L}(\mathcal{H})$ such $\pi(1)=1$ and $\pi\left(e^{i \theta}\right)=T$. To see that an $m$-isometry $T \in \mathcal{L}(\mathcal{H})$ does indeed have a $\mathcal{D}_{a}$ functional calculus, define $\pi: \mathcal{D}_{a} \rightarrow \mathcal{L}(\mathcal{H})$ by

$$
\pi(\varphi)=\sum_{k=0}^{\infty} \hat{\varphi}(k) T^{k}
$$

To see that (1.19) in fact defines a continuous algebra homomorphism it suffices to check that $\pi$ is well defined and continuous. Note that the topology on $\mathcal{D}_{a}$ is given by the seminorms

$$
\|\varphi\|_{\alpha}=\left(\sum_{k=0}^{\infty}(k+1)^{2 \alpha}|\hat{\varphi}(k)|^{2}\right)^{\frac{1}{2}}, \quad \alpha>0 .
$$

Also observe that if $\alpha>\frac{m}{2}$ and $T$ is an $m$-isometry, then

$$
\sum_{k=0}^{\infty}(k+1)^{-2 \alpha}\left\|s_{T}(k)\right\|<\infty
$$


since $s_{T}(k)$ is a polynomial of degree $\leq m-1$. Thus, if $\varphi \in \mathcal{D}_{a}$ and $\alpha>\frac{m}{2}$,

$$
\begin{aligned}
\left\|\sum_{k=0}^{\infty} \hat{\varphi}(k) T^{k}\right\| & \leq \sum_{k=0}^{\infty}|\hat{\varphi}(k)|\left\|T^{k}\right\| \\
= & \sum_{k=0}^{\infty}\left(k^{\alpha}|\hat{\varphi}(k)|\right)\left(k^{-\alpha}\left\|T^{k}\right\|\right) \\
& \leq\left\|\left(\frac{1}{i} \frac{d}{d \theta}\right)^{\alpha} \varphi\right\|_{2}\left(\sum_{k=0}^{\infty} k^{-2 \alpha}\left\|s_{T}(k)\right\|\right)^{\frac{1}{2}}
\end{aligned}
$$

so that $\pi$ is continuous. It is equally easy to define the analogous homomorphism of $\mathcal{D}$ in the case when $T$ is invertible. Summarizing we have established the following proposition.

Proposition 1.20. Let $T \in \mathcal{L}(\mathcal{H})$ be an $m$-isometry. $T$ has a $\mathcal{D}_{a}$-functional calculus. If $T$ is invertible, then $T$ has a $\mathcal{D}$-functional calculus.

We now describe the spectral picture of an $m$-isometry. If $T$ is an $m$-isometry, then $s_{T}(k)$ is a polynomial in $k$ and so the spectral radius formula implies that $\sigma(T) \subseteq \mathbb{D}^{-}$. The following lemma gives a more detailed description of the spectrum.

Lemma 1.21. If $T \in \mathcal{L}(\mathcal{H})$ is an $m$-isometry, then the approximate point spectrum lies in the unit circle. Thus, either $\sigma(T) \subseteq \partial \mathbb{D}$ or $\sigma(T)=\mathbb{D}^{-}$. In particular, $T$ is injective and $\operatorname{ran}(T)$ is closed.

Proof. If $\lambda \in \mathbb{C}$ is in the approximate point spectrum of $T$, then there exist unit vectors $x_{\ell} \in \mathcal{H}$ such that $(T-\lambda) x_{\ell} \rightarrow 0$. By induction, for each integer $k \geq 1,\left(T^{k}-\lambda^{k}\right) x_{\ell} \rightarrow 0$. Thus

$$
\begin{aligned}
0 & =\left\langle\left(\sum_{k=0}^{m}(-1)^{m-k}\left(\begin{array}{c}
m \\
k
\end{array}\right) T^{* k} T^{k}\right) x_{\ell}, x_{\ell}\right\rangle \\
& =\sum_{k=0}^{m}(-1)^{m-k}\left(\begin{array}{c}
m \\
k
\end{array}\right)\left\langle T^{k} x_{\ell}, T^{k} x_{\ell}\right\rangle \\
& \rightarrow \sum_{k=0}^{m}(-1)^{m-k}\left(\begin{array}{c}
m \\
k
\end{array}\right)|\lambda|^{2 k} \\
& =\left(|\lambda|^{2}-1\right)^{m}
\end{aligned}
$$

and so $|\lambda|=1$. Since $\partial \sigma(T) \subseteq \sigma_{a p}(T) \subseteq \partial \mathbb{D}, \sigma(T) \subseteq \partial \mathbb{D}$ or $\sigma(T)=\mathbb{D}^{-}$. Since $0 \notin \sigma_{a p}(\tau)$, $T$ is injective and $\operatorname{ran}(T)$ is closed. This establishes Lemma 1.21. 
Before continuing, we make the following remarks concerning the spectral picture of an $m$-isometry $T$. Fix $\lambda \in \mathbb{D}$. Since $\sigma_{\ell e}(T) \subseteq \sigma_{a p}(T) \subseteq \partial \mathbb{D}, T-\lambda$ is semi-Fredholm. Since $\sigma_{p}(T) \subseteq \partial \mathbb{D}$, the Fredholm index of $T-\lambda, \operatorname{ind}(T-\lambda)$, is nonpositive. Finally, since $\sigma_{\ell e}(T) \subseteq \partial \mathbb{D}$, the function from $\mathbb{D}$ into $\mathbb{Z} \cup\{ \pm \infty\}$ given by $\lambda \mapsto \operatorname{ind}(T-\lambda)$ is constant (See, for example, $[\mathbf{C}]$ ).

Corollary 1.22. Let $T \in \mathcal{L}(\mathcal{H})$. If both $T$ and $T^{*}$ are $m$-isometries, then $\sigma(T) \subseteq \partial \mathbb{D}$.

Proof. We argue by contradiction. By Lemma 1.21, if $\sigma(T) \nsubseteq \partial \mathbb{D}$, then $\sigma(T)=\mathbb{D}^{-}$. Since Lemma 1.21 also implies that $0 \notin \sigma_{a p}(T)$, we see that $(\operatorname{ran} T)^{-} \neq \mathcal{H}$. Hence $0 \in$ $\sigma_{a p}\left(T^{*}\right)$ contradicting Lemma 1.21 and the fact that $T^{*}$ is an $m$-isometry.

Thus, there are two cases: if $T$ is an $m$-isometry either $T$ is invertible and $\sigma(T) \subseteq \partial \mathbb{D}$ or $T$ is not invertible and $\sigma(T)=\mathbb{D}^{-}$. In fact both of these cases cannot occur for all $m$.

Proposition 1.23. If $T$ is an invertible $m$-isometry and $m$ is even, then $T$ is an $(m-1)$ isometry.

Proof. If $T$ is invertible, then one deduces as in the proof of Proposition 1.5 (let $k \rightarrow-\infty)$ that $-\triangle_{T} \geq 0$. Hence $\triangle_{T}=0$, i.e. $T$ is an $(m-1)$-isometry.

Note that Proposition 1.23 implies that every invertible 2-isometry is a unitary operator.

When applying the above observations to the case of 2 -isometries, one gains additional structure. If $\mathcal{H}$ is a Hilbert space and $S$ is a subset of $\mathcal{H}$, then we define $\vee S$ to be the smallest subspace of $\mathcal{H}$ which contains $S$ (i.e. $\vee S$ is the closed linear span of $S$ ). $T \in \mathcal{L}(\mathcal{H})$ is finitely cyclic if there exist an integer $n$ and there exist vectors $\gamma_{1}, \ldots, \gamma_{n} \in \mathcal{H}$ such that $\vee\left\{T^{k} \gamma_{j}: k \geq 0, \quad 1 \leq j \leq n\right\}=\mathcal{H}$.

Proposition 1.24. If $T \in \mathcal{L}(\mathcal{H})$ is a finitely cyclic 2-isometry, then $\triangle_{T}$ is compact.

Proof. Since $T$ is finitely cyclic, $T^{k}$ is finitely cyclic and so $\operatorname{ran}\left(T^{k}\right)^{\perp}$ is finite dimensional for every $k \geq 1$. In particular if $P_{k}$ denotes the orthogonal projection of $\mathcal{H}$ onto $\operatorname{ran}\left(T^{k}\right)$ : then $\triangle_{T}-P_{k} \triangle_{T} P_{k}$ is finite rank. Now fix $x \in \mathcal{H}$ and let $y=T^{k} x$. Since $T$ is a 2-isometry, $T^{*} \triangle_{T} T=\triangle_{T}$, and thus

$$
\begin{aligned}
\left\langle P_{k} \triangle_{T} P_{k} y, y\right\rangle & =\left\langle\triangle_{T} y, y\right\rangle \\
& =\left\langle\triangle_{T} T^{k} x, T^{k} x\right\rangle \\
& =\left\langle T^{* k} \triangle_{T} T^{k} x, x\right\rangle \\
& =\left\langle\triangle_{T} x, x\right\rangle .
\end{aligned}
$$


On the other hand, (1.3) implies that

$$
\left\langle\triangle_{T} x, x\right\rangle=\frac{1}{k}\left(\left\|T^{k} x\right\|^{2}-\|x\|^{2}\right) .
$$

Hence

$$
\begin{aligned}
\left\langle P_{k} \triangle_{T} P_{k} y, y\right\rangle= & \frac{1}{k}\left(\left\|T^{k} x\right\|^{2}-\|x\|^{2}\right) \\
& \leq \frac{1}{k}\left\|T^{k} x\right\|^{2} \\
& =\frac{1}{k}\|y\|^{2},
\end{aligned}
$$

and we see that $\left\|P_{k} \triangle_{T} P_{k}\right\| \rightarrow 0$. Hence

$$
\triangle_{T}=\lim _{k \rightarrow \infty}\left(\triangle_{T}-P_{k} \triangle_{T} P_{k}\right)
$$

is compact. This concludes the proof of Proposition 1.24.

In the case of 2-isometries Proposition 1.7 can be improved upon in that the subspace $\mathcal{M}$ of that proposition can be concretely identified.

Proposition 1.25. Let $T \in \mathcal{L}(\mathcal{H})$ be a 2-isometry and $\mathcal{N}$ be the smallest invariant subspace for $T$ generated by $\left(\operatorname{ran} \triangle_{T}\right)^{-} . \mathcal{N}$ reduces $T$ and $\mathcal{N}^{\perp}$ is the largest reducing subspace on which $T$ is isometric.

Proof. To show that $\mathcal{N}$ reduces $T$, it suffices to show that $\operatorname{ran}\left(T^{*} T^{n} \triangle_{T}\right) \subseteq \mathcal{N}$ for all $n=0,1,2, \ldots$

By Proposition 1.6, ker $\triangle_{T}$ is invariant for $T$ and so $\left(\operatorname{ran} \triangle_{T}\right)^{-}$is invariant for $T^{*}$. Thus $\operatorname{ran}\left(T^{*} \triangle_{T}\right) \subseteq\left(\operatorname{ran} \triangle_{T}\right)^{-} \subseteq \mathcal{N}$.

If $x \in \mathcal{H}$ and $n>0$, then

$$
\begin{aligned}
T^{*}\left(T^{n} \triangle_{T} x\right) & =T^{*} T\left(T^{n-1} \triangle_{T} x\right) \\
& =\left(\triangle_{T}+I\right)\left(T^{n-1} \triangle_{T} x\right) \\
& \in \operatorname{ran} \triangle_{T}+\operatorname{ran} T^{n-1} \triangle_{T} \\
& \subseteq \mathcal{N} .
\end{aligned}
$$

Thus $\mathcal{N}$ reduces $T$.

Suppose now that $\mathcal{N}_{0}$ reduces $T$ and $T \mid \mathcal{N}_{0}$ is isometric. By Proposition 1.6, $\mathcal{N}_{0} \subseteq$ ker $\triangle_{T}$ and so $\mathcal{N}_{0}^{\perp} \supseteq \operatorname{ran} \triangle_{T}$. Since $\mathcal{N}_{0}^{\perp}$ is invariant for $T, \mathcal{N}_{0}^{\perp} \supseteq \mathcal{N}$. Thus $\mathcal{N}_{0} \subseteq \mathcal{N}^{\perp}$. This establishes Proposition 1.25.

We close this section with a decomposition theorem for 2 -isometries that contains both the decompositions of (1.15) and (1.18). 
Theorem 1.26. Let $T \in \mathcal{L}(\mathcal{H})$ be a 2-isometry. If $\mathcal{M}$ is the largest reducing subspace for $T$ on which $T$ is isometric then $T$ has the block representation

$$
T=\left[\begin{array}{ccc}
V_{0} & 0 & 0 \\
0 & V & E \\
0 & 0 & X
\end{array}\right]
$$

with respect to the decomposition,

$$
\mathcal{H}=\mathcal{M} \oplus\left(\operatorname{ker} \Delta_{T} \ominus \mathcal{M}\right) \oplus\left(\operatorname{ran} \Delta_{T}\right)^{-}
$$

Furthermore,

$$
V_{0} \text { is an isometry, }
$$

$$
V \text { is a pure isometry, }
$$

and

$$
\begin{gathered}
V^{*} E=0, \\
X^{*}\left(E^{*} E+X^{*} X-1\right) X=E^{*} E+X^{*} X-1, \\
\operatorname{ran} E \text { is dense in } \operatorname{ker} V^{*},
\end{gathered}
$$

Proof. By Proposition 1.6, ker $\triangle_{T}$ is invariant for $T$ and $\mathcal{M} \subseteq \operatorname{ker} \triangle_{T}$. Hence $T$ has the form of (1.27) with respect to the decomposition (1.28) and (1.29) holds. Computing using (1.27) one finds that

$$
\triangle_{T}=\left[\begin{array}{ccc}
0 & 0 & 0 \\
0 & V^{*} V-1 & V^{*} E \\
0 & E^{*} V & E^{*} E+X^{*} X-1
\end{array}\right] .
$$

Hence $V$ is an isometry, and (1.31) and (1.34) hold. Computing

$$
T^{*} \triangle_{T} T=\Delta_{T}
$$


yields that (1.32) holds. Now observe that Proposition 1.25 implies that

$$
\vee\left\{V^{k} \operatorname{ran} E: k \geq 0\right\} \text { is dense in } \text { ker } \triangle_{T} \ominus \mathcal{M} .
$$

Since $V$ is an isometry and $\operatorname{ran} E$ is in the initial space of $V$ (i.e. ran $E \subseteq$ ker $\left.V^{*}\right),(1.35)$ immediately implies that $V$ is pure and $\operatorname{ran} E$ is dense in ker $V^{*}$. This concludes the proof of Theorem 1.26.

We observe in Theorem 1.26 that if $T$ is a 2 -isometry that has the representation of (1.27) but with respect to a decomposition

$$
\mathcal{H}=\mathcal{H}_{0} \oplus \mathcal{H}_{1} \oplus \mathcal{H}_{2}
$$

and with (1.29)-(1.34) holding then necessarily $\mathcal{H}_{0}=\mathcal{M}$, the largest reducing subspace for $T$ such $T \mid \mathcal{M}$ is isometric, $\mathcal{H}_{1}=$ ker $\triangle_{T} \ominus \mathcal{M}$, and $\mathcal{H}_{2}=\left(\operatorname{ran} \triangle_{T}\right)^{-}$.

In the case of isometries one can do much better than Theorem 1.26 in that one can through abstract arguments $([\mathbf{F}])$ explicitly display the general pure isometry as a direct sum of cyclic pure isometries. That such an approach will not be successful for 2-isometries is seen by the following example of a noncyclic 2-cyclic pure 2-isometry:

$$
\left[\begin{array}{cccc}
S & 0 & \sqrt{2} \otimes 1 & 0 \\
0 & S & 0 & \sigma \otimes 1 \\
0 & 0 & 1 & b \\
0 & 0 & 0 & -1
\end{array}\right] .
$$

\section{§2. Distribution Differential Operators on the circle}

Let $\mathcal{D}=C^{\infty}(\partial \mathbb{D})$, the Frechet space of infinitely differentiable functions on the unit circle. Let $\mathcal{D}^{\prime}$ denote the dual of $\mathcal{D}$, the space of distributions on the circle.

We define a linear operator $D: \mathcal{D} \rightarrow \mathcal{D}$ via the formula

$$
D \varphi=\frac{1}{i} \frac{d}{d \theta} \varphi
$$

If $\ell \geq 0$ is an integer, we define $D^{(\ell)}: \mathcal{D} \rightarrow \mathcal{D}$ by

$$
D^{(\ell)}=D \cdot(D-1) \cdots(D-\ell+1)
$$

Recalling that $e^{i k \theta} \in \mathcal{D}$ is an eigenfunction for $D$ with corresponding eigenvalue $k$, we see that

$$
D^{(\ell)}\left(e^{i k \theta}\right)=k^{(\ell)} e^{i k \theta},
$$

a formula which we use repeatedly in the sequel.

Now recall that if $\beta \in \mathcal{D}^{\prime}$ and $\varphi \in \mathcal{D}$ then $\varphi \beta \in \mathcal{D}^{\prime}$ can be defined by

$$
\varphi \beta(\psi)=\beta(\varphi \psi), \quad \psi \in \mathcal{D}
$$


Thus, if $\beta \in \mathcal{D}^{\prime}, \beta$ can be regarded naturally as a map $\beta: \mathcal{D} \rightarrow \mathcal{D}^{\prime}$ by defining

$$
\beta(\varphi)=\varphi \beta .
$$

With the notation of the preceding two paragraphs, we are now able to define the class of objects that will be the subject of this section.

Definition 2.1. A distribution differential operator DDO is a map $L: \mathcal{D} \rightarrow \mathcal{D}^{\prime}$ that has the form

$$
L=\sum_{\ell=0}^{m} \beta_{\ell} D^{(\ell)}
$$

where $\beta_{0}, \ldots, \beta_{m} \in \mathcal{D}^{\prime}$. If $L$ is as in (2.2) and $\beta_{m} \neq 0$, we say $L$ has order $m$.

If $L$ is a DDO and $\varphi, \psi \in \mathcal{D}$, let us agree to define $\psi L \varphi: \mathcal{D} \rightarrow \mathcal{D}^{\prime}$ by

$$
(\psi L \varphi)(\chi)=\psi L(\varphi \chi), \quad \chi \in \mathcal{D}
$$

With this definition observe that if $L$ is a DDO of order $m$ and $\varphi, \psi \in \mathcal{D}$, then $\psi L \varphi$ is a DDO of order $\leq m$. As a final piece of notation before stating our first lemma, we introduce the formal derivative of a DDO.

Definition 2.3. If $L=\sum \beta_{\ell} D^{(\ell)}$ is a DDO of order $m$, we define the DDO $\frac{d}{d D} L$ of order $m-1$ by setting

$$
\frac{d}{d D} L=\sum \ell \beta_{\ell} D^{(\ell-1)}
$$

The following lemma is a key piece of algebra that not only will lead to several characterizations of DDO, but will also allow us in Section 3 to characterize positive definite DDO as the key analytical objects corresponding to the model theory of $m$-isometries.

Lemma 2.4. If $L$ is a $D D O$, then

$$
e^{-i \theta} L e^{i \theta}-L=\frac{d}{d D} L
$$

Proof. It suffices to establish the formula for the case $L=\beta D^{(\ell)}$. We observe that Leibnitz's rule is true for $D^{(\ell)}$ in the form:

$$
D^{(\ell)}(\varphi \psi)=\sum_{j=0}^{\ell}\left(\begin{array}{l}
\ell \\
j
\end{array}\right) D^{(j)} \varphi D^{(\ell-j)} \psi .
$$

Thus, using the fact that $D^{(j)}\left(e^{i \theta}\right)=0$ if $j>1$,

$$
\begin{aligned}
\left(e^{-i \theta}\left(\beta D^{(\ell)}\right) e^{i \theta}-\beta D^{(\ell)}\right)(\varphi) & =e^{-i \theta} \beta\left(e^{i \theta} D^{(\ell)} \varphi+\ell e^{i \theta} D^{(\ell-1)} \varphi\right)-\beta\left(D^{(\ell)} \varphi\right) \\
& =\ell \beta D^{(\ell-1)}(\varphi) .
\end{aligned}
$$

This establishes Lemma 2.4.

We obtain the following immediate corollary. 
Lemma 2.5. If $L$ is a $D D O$ and $m \geq 0$, then

$$
\left.\delta^{m}\left\{L\left(e^{i k \theta} \varphi\right)\left(e^{-i k \theta} \bar{\psi}\right)\right\}\right|_{k=0}=\left(\frac{d}{d D}\right)^{m} L(\varphi)(\bar{\psi})
$$

Proposition 2.7. If $\left\{a_{k_{1}, k_{2}}\right\}$ is bilateral, temperate and polynomial of degree $m$ on diagonals, then there exists a unique DDO $L$ of order $m$ such that

$$
a_{k_{1}, k_{2}}=L\left(e^{i k_{1} \theta}\right)\left(e^{-i k_{2} \theta}\right), \quad k_{1}, k_{2}, \in \mathbb{Z}
$$

Conversely, if $L$ is a DDO of order $m$ and $\left\{a_{k_{1}, k_{2}}\right\}$ is defined as in (2.8), then $\left\{a_{k_{1}, k_{2}}\right\}$ is bilateral, temperate and polynomial of degree $m$ on diagonals.

Proof. Let $\left\{a_{k_{1}, k_{2}}\right\}$ be bilateral, temperate and polynomial of degree $m$ on diagonals. If $k \in \mathbb{Z}$ is fixed, then $a_{j, k+j}$ is a polynomial in $j$ of degree $\leq m$. Thus, there exist $b_{\ell, k} \in \mathbb{C}$ for $\ell=0,1, \ldots, m$ such that

$$
a_{j, k+j}=\sum_{\ell=0}^{m} b_{\ell, k} j^{(\ell)}, \quad j \in \mathbb{Z}
$$

Since $\left\{a_{k_{1}, k_{2}}\right\}$ is temperate, we can define a distribution $\beta_{\ell}$ satisfying

$$
\hat{\beta}_{\ell}(k)=b_{\ell, k}
$$

Computing, we find that if $L$ is defined by

$$
L=\sum_{\ell=0}^{m} \beta_{\ell} D^{(\ell)}
$$

then

$$
\begin{aligned}
L\left(e^{i j \theta}\right)\left(e^{-i(k+j) \theta}\right) & =\sum_{\ell} \beta_{\ell}\left(\left(D^{(\ell)} e^{i j \theta}\right)\left(e^{-i(k+j) \theta}\right)\right) \\
& =\sum_{\ell} j^{(\ell)} \beta_{\ell}\left(e^{-i k \theta}\right) \\
& =\sum_{\ell} b_{\ell, k} j^{(\ell)} \\
& =a_{j, k+j} .
\end{aligned}
$$

To see that $L$ is unique, simply observe that if $L=\sum_{\ell=0}^{m} \beta_{\ell} D^{(\ell)}$ is any DDO such that (2.8) holds, then (2.9) holds. 
Conversely, let $L$ be a DDO of order $m$ and $\left\{a_{k_{1}, k_{2}}\right\}$ be defined as in (2.8). For fixed $k_{1}, k_{2} \in \mathbb{Z}$, we define a sequence $\left\{b_{j}\right\}$ by

$$
b_{j}=a_{k_{1}+j, k_{2}+j,}, \quad j \in \mathbb{N} .
$$

Note that Lemma 2.5 implies that

$$
\begin{aligned}
\left(\delta^{m+1} b\right)_{j} & =\left(\frac{d}{d D}\right)^{m+1} L\left(e^{i\left(k_{1}+j\right) \theta}\right)\left(e^{-i\left(k_{2}+j\right) \theta}\right) \\
& =0
\end{aligned}
$$

since $L$ has order $m$. Hence $\left\{a_{k_{1}, k_{2}}\right\}$ is polynomial of degree $m$ on diagonals. Finally, if $L=\sum \beta_{\ell} D^{(\ell)}$, then

$$
\begin{aligned}
a_{k_{1}, k_{2}} & =\sum \beta_{\ell}\left(\left(D^{(\ell)} e^{i k_{1} \theta}\right) e^{-k_{2} \theta}\right) \\
& =\sum k_{1}^{(\ell)} \beta_{\ell}\left(e^{i\left(k_{1}-k_{2}\right) \theta}\right) \\
& =\sum k_{1}^{(\ell)} \hat{\beta}_{\ell}\left(k_{2}-k_{1}\right)
\end{aligned}
$$

and so $\left\{a_{k_{1}, k_{2}}\right\}$ is temperate. This establishes Proposition 2.7 .

The following corollary is the unilateral version of Proposition 2.7.

Corollary 2.10. If $\left\{a_{k_{1}, k_{2}}\right\}$ is unilateral, temperate and polynomial of degree $m$ on diagonals, then there exists a unique DDO $L$ of order $m$ such that

$$
a_{k_{1}, k_{2}}=L\left(e^{i k_{1} \theta}\right)\left(e^{-i k_{2} \theta}\right) \quad k_{1}, k_{2} \geq 0 .
$$

Conversely, if $L$ is a DDO of order $m$ and $\left\{a_{k_{1}, k_{2}}\right\}$ is defined as in (2.11), then $\left\{a_{k_{1}, k_{2}}\right\}$ is unilateral, temperate and polynomial of degree $m$ on diagonals.

ProOf. The converse follows immediately from Proposition 2.7. If $\left\{a_{k_{1}, k_{2}}\right\}$ is unilateral and polynomial of degree $m$ on diagonals, then there is a unique bilateral array $\left\{b_{k_{1}, k_{2}}\right\}$ which extends $\left\{a_{k_{1}, k_{2}}\right\}$ (i.e., $b_{k_{1}, k_{2}}=a_{k_{1}, k_{2}}, k_{1}, k_{2} \geq 0$ ) and is polynomial of degree $m$ on diagonals. Furthermore, if $\left\{a_{k_{1}, k_{2}}\right\}$ is temperate, then $\left\{b_{k_{1}, k_{2}}\right\}$ is temperate. By Proposition 2.7 , there exists a unique DDO $L$ satisfying

$$
b_{k_{1}, k_{2}}=L\left(e^{i k, \theta}\right)\left(e^{-i k_{2} \theta}\right) \quad k_{1}, k_{2} \in \mathbb{Z} .
$$

Thus (2.11) holds. Since $\left\{a_{k_{1}, k_{2}}\right\}$ has a unique extension to a bilateral array and Proposition 2.17 guarantees a unique DDO $L$, the DDO $L$ satisfying (2.11) is unique. This establishes Corollary 2.10 .

We record for future reference the following formula which is an immediate consequence of Lemma 2.5 and Proposition 2.7 (which asserts that $\left(e^{-i k \theta} \operatorname{Le} e^{i k \theta}\right)(\varphi)(\vec{\psi})$ is a polynomial in $k$ ). 
Lemma 2.12. Let $L=\sum_{\ell=0}^{m} \beta_{\ell} D^{(\ell)}$ be a DDO. If $\varphi$ and $\psi$ are in $\mathcal{D}$, then

$$
\lim _{k \rightarrow \infty} \frac{1}{k^{(m)}}\left(e^{-i k \theta} L e^{i k \theta}\right)(\varphi)(\bar{\psi})=\beta_{m}(\varphi \bar{\psi})
$$

The Schwarz nuclear theorem guarantees that there is a one-to-one correspondence between the continuous linear maps $L: \mathcal{D} \rightarrow \mathcal{D}^{\prime}$ and the distributions $u$ on the torus, $(\partial \mathrm{D})^{2}$. This correspondence is implemented by

$$
u\left(\varphi\left(e^{i \theta_{1}} \overline{\psi\left(e^{i \theta_{2}}\right.}\right)\right)=L(\varphi)(\bar{\psi})
$$

Proposition 2.7 and Corollary 2.10 give a characterization of DDO inside the space of continuous linear maps $L$ from $\mathcal{D}$ to $\mathcal{D}^{\prime}$ in terms of the Fourier transform of the corresponding distributions $u$. (If (2.8) and (2.13) hold, then $\left.\hat{u}\left(-k_{1}, k_{2}\right)=a_{k_{1}, k_{2}}\right)$. Another characterization of DDO is provided by the following proposition.

Proposition 2.14. The following conditions on a continuous linear mapping $L: \mathcal{D} \rightarrow \mathcal{D}^{\prime}$ are equivalent.

(i) There exists an $m \geq 0$ such that

$$
\sum_{\ell=0}^{m}(-1)^{m-\ell}\left(\begin{array}{c}
m \\
\ell
\end{array}\right) e^{-i \ell \theta} L e^{i \ell \theta}=0
$$

(ii) $L$ is a DDO.

(iii) The distribution $u$ defined by (2.13) has support in the set $\left\{\left(e^{i \theta}, e^{i \theta}\right): \theta \in \mathbb{R}\right\}$.

Proof. Observe that (i) implies that if the bilateral array $\left\{a_{k_{1}, k_{2}}\right\}$ is defined by

$$
a_{k_{1}, k_{2}}=L\left(e^{i k_{1} \theta}\right)\left(e^{-i k_{2} \theta}\right)
$$

then $\left\{a_{k_{1}, k_{2}}\right\}$ is polynomial on diagonals. $\left\{a_{k_{1}, k_{2}}\right\}$ is temperate since $L$ is continuous. By Proposition 2.7, there exists a DDO $L_{0}$ such that

$$
a_{k_{1}, k_{2}}=L_{0}\left(e^{i k_{1} \theta}\right)\left(e^{-i k_{2} \theta}\right) .
$$

If $k_{1}$ is fixed, then (2.15) and (2.16) imply that

$$
L_{0}\left(e^{i k_{1} \theta}\right) \hat{}\left(k_{2}\right)=L\left(e^{i k_{1} \theta}\right) \hat{(}\left(k_{2}\right)
$$

for all $k_{2} \in \mathbb{Z}$. Hence

$$
L_{0}\left(e^{i k_{1} \theta}\right)=L\left(e^{i k_{1} \theta}\right)
$$


Since $k_{1}$ is arbitrary and $L$ is continuous, (2.12) implies that $L=L_{0}$. Thus, $L$ is a DDO. This shows that (i) implies (ii).

Suppose (ii) holds and $u$ is defined by (2.13). If $m$ is the order of $L$, then

$$
\begin{aligned}
u\left(\left(1-e^{i\left(\theta_{1}-\theta_{2}\right)}\right)^{m} \varphi\left(e^{i \theta_{1}} \overline{\psi\left(e^{i \theta_{2}}\right.}\right)\right) & \left.=\sum_{k=0}^{m}(-1)^{k}\left(\begin{array}{c}
m \\
k
\end{array}\right) u\left(e^{i k \theta_{1}} \varphi\left(e^{i \theta_{1}} \overline{e^{i k \theta_{2}} \psi\left(e^{i \theta_{2}}\right.}\right)\right)\right) \\
& =\sum_{k=0}^{m}(-1)^{k}\left(\begin{array}{c}
m \\
k
\end{array}\right) L\left(e^{i k \theta} \varphi\right)\left(e^{-i k \theta} \bar{\psi}\right) \\
& =\left(\frac{d}{d D}\right)^{m+1} L(\varphi)(\bar{\psi}) \\
& =0 .
\end{aligned}
$$

Thus, (iii) holds.

Suppose now that (iii) holds. Since $(\partial D)^{2}$ is compact, $u$ has finite order, and thus, there exists an $m \geq 0$ such that $\left(1-e^{i\left(\theta_{1}-\theta_{2}\right)}\right)^{m} u=0$. For $\varphi, \psi \in \mathcal{D}$,

$$
\begin{aligned}
\left(\sum_{\ell=0}^{m}(-1)^{m-\ell}\left(\begin{array}{c}
m \\
\ell
\end{array}\right) e^{-i \ell \theta} L e^{i \ell \theta}\right)(\varphi)(\bar{\psi}) & \left.=\sum_{\ell=0}^{m}(-1)^{m-\ell}\left(\begin{array}{c}
m \\
\ell
\end{array}\right) L\left(e^{i \ell \theta} \varphi\right) \overline{\left(e^{i \ell \theta} \psi\right.}\right) \\
& =\sum_{\ell=0}^{m}(-1)^{m-\ell}\left(\begin{array}{c}
m \\
\ell
\end{array}\right) u\left(e^{i \ell \theta_{1}} \varphi \overline{e^{i \ell \theta_{2}} \psi}\right) \\
& =u\left(\left(1-e^{i\left(\theta_{1}-\theta_{2}\right)}\right)^{m} \varphi \bar{\psi}\right) \\
& =0 .
\end{aligned}
$$

Thus (i) holds. This establishes Proposition 2.14.

\section{§3. A model theory for $m$-isometries}

In this section, we shall model the general finitely cyclic $m$-isometry as multiplication by $z$ on the completion of a pre-Hilbert space of analytic functions with respect to a norm defined by a DDO.

Define $\mathcal{D}_{a} \subset \mathcal{D}$ by

$$
\mathcal{D}_{a}=\{\varphi \in \mathcal{D}: \hat{\varphi}(n)=0 \text { if } n<0\} .
$$

Since the Fourier transform theory implies that $\varphi \in \mathcal{D}_{a}$ if and only if

$$
\varphi\left(e^{i \theta}\right)=\sum_{n=0}^{\infty} \hat{\varphi}(n) e^{i n \theta}
$$

with convergence in $\mathcal{D}$, it is clear that $\mathcal{D}_{a}$ consists precisely of the boundary values of analytic functions on $\mathbb{D}$ whose power series coefficients form a rapidly decreasing sequence. We let $P$ denote the canonical projection of $\mathcal{D}$ onto $\mathcal{D}_{a}$ defined by

$$
(P \varphi)^{n}(n)=\left\{\begin{array}{cc}
\varphi(n) & n \geq 0 \\
0 & n<0
\end{array}\right.
$$


In like fashion, let $\mathcal{D}_{a}^{\prime}$ denote the space of analytic distributions defined by

$$
\mathcal{D}_{a}^{\prime}=\left\{u \in \mathcal{D}^{\prime}: \hat{u}(n)=0 \text { if } n<0\right\}
$$

$\mathcal{D}_{a}^{\prime}$ can be regarded as the space of boundary values of analytic functions on $\mathbb{D}$ whose power series coefficients form a temperate sequence. We let $P$ denote the canonical projection of $\mathcal{D}^{\prime}$ onto $\mathcal{D}_{a}^{\prime}$ defined by

$$
(P u) \hat{(n)}=\left\{\begin{array}{cc}
\hat{u}(n) & n \geq 0 \\
0 & n<0
\end{array} .\right.
$$

Definition 3.1. A distribution Toeplitz operator (DTO) is a linear mapping $A: \mathcal{D}_{a} \rightarrow \mathcal{D}_{a}^{\prime}$ that has the form

$$
A=P L \mid \mathcal{D}_{a}
$$

for some DDO $L$. If $A$ is a DTO, we define the order of $A$ to be the order of $L$ where $L$ is as in (3.2).

Lemma 3.3. If $A$ and $L$ are as in (3.2), then

$$
A(\varphi)(\bar{\psi})=L(\varphi)(\bar{\psi})
$$

whenever $\varphi, \psi \in \mathcal{D}_{a}$.

Proof. We first show that if $u \in \mathcal{D}^{\prime}$, then

$$
P u(\bar{\psi})=u(\bar{\psi})
$$

whenever $\psi \in \mathcal{D}_{a}$. Accordingly, fix $u \in \mathcal{D}^{\prime}$ and $\psi \in \mathcal{D}_{a}$.

$$
\begin{aligned}
P u(\bar{\psi}) & =\left(\sum_{k=0}^{\infty} \hat{u}(k) e^{i k \theta} \frac{d \theta}{2 \pi}\right)\left(\sum_{k=0}^{\infty} \overline{\hat{\psi}(k)} e^{-i k \theta}\right) \\
& =\sum_{k=0}^{\infty} \hat{u}(k) \overline{\hat{\psi}(k)} \\
& =\sum_{k=-\infty}^{\infty} \hat{u(k)} \overline{\hat{\psi}(k)} \\
& =u(\bar{\psi}) .
\end{aligned}
$$

Using (3.4), we now see that

$$
\begin{aligned}
A(\varphi)(\bar{\psi}) & =P(L(\varphi))(\bar{\psi}) \\
& =L(\varphi)(\bar{\psi})
\end{aligned}
$$


which establishes Lemma 3.3 .

As a corollary to Lemma 3.3 and Corollary 2.10, one sees that if $A$ is a DTO, then the DDO $L$ satisfying (3.2) is unique. In particular, the notion of the order of $A$ in Definition 3.1 is well-defined.

If $L$ is a DDO, let us agree to say that $L$ is positive $(L \geq 0)$ if

$$
L(\varphi)(\ddot{\varphi}) \geq 0
$$

whenever $\varphi \in \mathcal{D}$. If $A$ is a DTO, let us agree to say that $A$ is positive $(A \geq 0)$ if

$$
A(\varphi)(\bar{\varphi}) \geq 0
$$

whenever $\varphi \in \mathcal{D}_{a}$. We remark before continuing that if $L$ is a $D D O$ and $A=P L \mid \mathcal{D}_{a}$ is a DTO, then it is possible that $A \geq 0$ yet $L$ is not positive. An example is given by letting $L=D$. This is a reflection of the operator theoretic fact that unlike the case when $m=1$, not all $m$-isometries have extensions to invertible $m$-isometries.

Lemma 3.5. Let $L=\sum_{\ell=0}^{m} \beta_{\ell} D^{(\ell)}$ be a $D D O$ with $\beta_{m} \neq 0$. If $L \geq 0$, then $\beta_{m}$ is a positive measure. If $A=P L \mid \mathcal{D}_{a} \geq 0$, then $\beta_{m}$ is a positive measure.

Proof. If $L \geq 0$, then $A \geq 0$. Thus, it suffices to show only the final assertion of the lemma. Thus, assume $A \geq 0$. Fix $\varphi \in \mathcal{D}_{a}$. By Lemma 2.12,

$$
\begin{aligned}
\beta_{m}\left(|\varphi|^{2}\right) & =\lim _{k \rightarrow \infty} \frac{1}{k^{(m)}}\left(e^{-i k \theta} L e^{i k \theta}\right)(\varphi)(\bar{\varphi}) \\
& =\lim _{k \rightarrow \infty} \frac{1}{k^{(m)}} A\left(e^{i k \theta} \varphi\right)\left(\overline{e^{i k \theta} \varphi}\right) \\
& \geq 0
\end{aligned}
$$

Hence,

$$
\beta_{m}\left(|\varphi|^{2}\right) \geq 0 \quad \text { whenever } \quad \varphi \in \mathcal{D}_{a}
$$

But,

$$
\left\{|\varphi|^{2}: \varphi \in \mathcal{D}_{a}\right\} \text { is dense in }\{\psi \in \mathcal{D}: \psi \geq 0\}
$$

Hence (3.6) implies that $\beta_{m}$ is a positive distribution and thus is a positive measure. This concludes the proof of Lemma 3.5.

If $L \geq 0$ is a DDO, we define the form space of $L, H_{L}^{2}$, to be the completion of $\mathcal{D}$ with respect to the sesquilinear form $[\cdot, \cdot]_{L}$ on $\mathcal{D} \times \mathcal{D}$ given by

$$
[\varphi, \psi]=L(\varphi)(\bar{\psi})
$$


Specifically, one sets $\mathcal{M}_{L}=\left\{\varphi \in \mathcal{D}:[\varphi, \varphi]_{L}=0\right\}$, observes by the Cauchy-Schwarz inequality that $[\cdot, \cdot]_{L}$ induces a inner product on $\mathcal{D} / \mathcal{M}_{L}$, and lets $H_{L}^{2}$ denote the completion of $\mathcal{D} / \mathcal{M}_{L}$.

Likewise, if $A \geq 0$ is a DTO, we define the form space of $A, H_{A}^{2}$, to be the completion of $\mathcal{D}_{a}$ with respect to the sesquilinear form $[\cdot, \cdot]_{A}$ on $\mathcal{D}_{a} \times \mathcal{D}_{a}$ given by

$$
[\varphi, \psi]_{A}=A(\varphi)(\bar{\psi})
$$

If $L \geq 0$ is a DDO, then one can attempt to define a linear operator $M_{L}$ on $H_{L}^{2}$ via the formula

$$
\left(M_{L} \varphi\right)\left(e^{i \theta}\right)=e^{i \theta} \varphi\left(e^{i \theta}\right)
$$

In general, this operator need not be well-defined on $\mathcal{D} / \mathcal{M}_{L}$ and, even if it is, may not extend to a bounded operator on $H_{L}^{2}$.

Lemma 3.7. Let $L$ be a $D D O$ and fix $c>1 . L \geq 0$ and $M_{L}$ is a well-defined bounded operator on $H_{L}^{2}$ of norm $\leq c$ if and only if

$$
0 \leq L-c^{-2} e^{-i \theta} L e^{i \theta}
$$

Proof. First assume that $L \geq 0$ and $\left\|M_{L}\right\| \leq c$. If $\varphi \in \mathcal{D}$, then

$$
\begin{aligned}
\left(L-c^{-2} e^{-i \theta} L e^{i \theta}\right)(\varphi)(\bar{\varphi}) & =L(\varphi)(\bar{\varphi})-c^{-2} L\left(e^{i \theta} \varphi\right)\left(\overline{e^{i \theta} \varphi}\right) \\
& =\|\varphi\|_{L}^{2}-c^{-2}\left\|M_{L} \varphi\right\|_{L}^{2} \\
& \geq 0
\end{aligned}
$$

and thus (3.8) holds. Conversely, assume that $L$ is a DDO satisfying (3.8). Note that by conjugation each term in the following telescoping sum is positive:

$$
L-c^{-2 k} e^{-i k \theta} L e^{i k \theta}=\sum_{j=0}^{k-1} c^{-2 j} e^{-i j \theta}\left(L-c^{-2} e^{-i \theta} L e^{i \theta}\right) e^{i j \theta}
$$

Also note by Proposition 2.7 that

$$
\left(e^{-i k \theta} L e^{i k \theta}\right)(\varphi)(\bar{\varphi})=(\bar{\varphi} L \varphi)\left(e^{i k \theta}\right)\left(e^{-i k \theta}\right)
$$

is a polynomial in $k$. Hence since $c>1$

$$
\lim _{k \rightarrow \infty} c^{-2 k} e^{-i k \theta} L e^{i k \theta}(\varphi)(\bar{\varphi})=0 .
$$


Consequently,

$$
\begin{aligned}
L(\varphi)(\bar{\varphi})= & \lim _{k \rightarrow \infty}\left(L-c^{-2 k} e^{-i k \theta} L e^{i k \theta}\right)(\varphi)(\bar{\varphi}) \\
& \geq 0
\end{aligned}
$$

and we have established that $L \geq 0$. Calculating as before establishes that

$$
\begin{aligned}
\|\varphi\|_{L}^{2}-c^{-2}\left\|e^{i \theta} \varphi\right\|_{L}^{2}= & \left(L-c^{-2} e^{-i \theta} L e^{i \theta}\right)(\varphi)(\bar{\varphi}) \\
& \geq 0 .
\end{aligned}
$$

whenever $\varphi \in \mathcal{D}$. This inequality establishes both that $M_{L}$ is well-defined on $\mathcal{D} / \mathcal{M}_{L}$ and extends by continuity to an operator $M_{L}$ on $H_{L}^{2}$ with $\left\|M_{L}\right\| \leq c$. This establishes Lemma 3.7.

Lemma 3.7 suggests that we introduce the following set for a DDO $L \neq 0$.

$$
S_{L}=\left\{c>0: 0 \leq L-c^{-2} e^{-i \theta} L e^{i \theta}\right\}
$$

There are three possibilities which we shall consider in succession.

Case 1. $S_{L}=\phi$. In this case, Lemma 3.7 guarantees that even though $L$ might be positive (so that $H_{L}^{2}$ is defined), either $M_{L}$ is ill defined on $\mathcal{D} / \mathcal{M}_{L}$ or does not extend by continuity to a bounded operator on $H_{L}^{2}$.

Case 2. $S_{L} \neq \phi$ and $\inf S_{L}>1$. In this case Lemma 3.7 guarantees that $L \geq 0$ and $M_{L}$ is a well-defined operator with

$$
\left\|M_{L}\right\|=\inf S_{L} .
$$

Note also that if ord $(L)=0$, then (3.6) holds with $c=1$ (i.e. $1 \in S_{L}$ ). Thus ord $(L) \geq 1$ in this case.

Case 3. $S_{L} \neq \phi$ and inf $S_{L} \leq 1$. In this case necessarily if $L \geq 0$, then ord $(L)=0$. To see this fix $c \leq 1$ with $c \in S_{L}$, and observe that if $k \geq 0$, then

$$
L-c^{-2 k} e^{-i k \theta} L e^{i k \theta}=\sum_{j=0}^{k-1} c^{-2 j} e^{-i j \theta}\left(L-c^{-2} e^{-i \theta} L e^{i \theta}\right) e^{i j \theta} \geq 0
$$

Hence if $\varphi \in \mathcal{D}$,

$$
L(\varphi)(\bar{\varphi})-c^{-2 k}\left(e^{-i k \theta} L e^{i k \theta}\right)(\varphi)(\bar{\varphi}) \geq 0 .
$$

Now if $m \geq 1$, then

$$
\lim _{k \rightarrow \infty} \frac{1}{k^{(m)}} L(\varphi)(\bar{\varphi})=0 .
$$


Hence by (3.9) and Lemma 2.12, if $L=\sum_{\ell} \beta_{\ell} D^{(\ell)}$, then

$$
-\beta_{m}(\varphi)(\bar{\varphi}) \geq 0
$$

But since $L \geq 0$, Lemma 3.5 implies that $\beta_{m} \geq 0$. Thus, $\beta_{m}=0$ contradicting $m \geq 1$.

We formalize the above observations into the following definition which precisely identifies those DDO $L$ with the property that $L \geq 0$ and $M_{L}$ is a well-defined bounded operator.

Definition 3.10. A Dirichlet operator is a DDO $L$ with the property that either there exists a constant $c>1$ such that

$$
0 \leq L-c^{-2} e^{-i \theta} L e^{i \theta}
$$

or ord $(L)=0$ and $L \geq 0$.

Note that in Definition 3.10, the condition ord $L=0$ and $L \geq 0$ is equivalent to the assertion that $L$ is a positive measure.

If $c$ is a given constant, then the Dirichlet operators $L$ with $\left\|M_{L}\right\| \leq c$ can be completely parameterized via an affine transformation by the positive DDO as described in the following proposition.

Proposition 3.11. The Dirichlet operators of order 0 consist of the positive measures. If $m \geq 1$, the Dirichlet operators with ord $(L)=m$ and $\left\|M_{L}\right\| \leq c$ consist precisely of the set

$$
\left\{\sum_{k=0}^{\infty} c^{-2 k} e^{-i k \theta} Q e^{i k \theta}: Q \quad \text { is a } \quad \text { DDO, } \text { ord }(Q)=m, Q \geq 0\right\}
$$

Proof. The first assertion of the proposition follows from the definition of Dirichlet operators. To prove the second assertion, note that the definition implies that if $L$ is a DDO and ord $(L) \geq 1$, then $\left\|M_{L}\right\|>1$. Hence it suffices to assume $c>1$. We first note that if $Q$ is a DDO and $c>1$, then the series

$$
\sum_{k=0}^{\infty} c^{-2 k} e^{-i k \theta} Q e^{i k \theta}
$$

converges. This guarantees that the following calculations are justified.

First assume that $L$ is a Dirichlet operator with ord $(L)=m$ and $\left\|M_{L}\right\| \leq c$. If $Q$ is defined by

$$
Q=L-c^{-2} e^{-i \theta} L e^{i \theta}
$$


then $Q$ is a DDO, ord $Q=m$ and $Q \geq 0$. Conjugating (3.12) by $c^{-k} e^{i k \theta}$ and summing in $k$ yields that

$$
\sum_{k=0}^{j-1} c^{-2 k} e^{-i k \theta} Q e^{i k \theta}=L-c^{-2 j} e^{-i j \theta} L e^{i j \theta} .
$$

Since $L$ is a DDO and $c>1$, Proposition 2.7 implies that

$$
\lim _{j \rightarrow \infty} c^{-2 j} e^{-i j \theta} L e^{i j \theta}=0
$$

Hence,

$$
L=\sum_{k=0}^{\infty} c^{-2 k} e^{-i k \theta} Q e^{i k \theta}
$$

Conversely, assume that $Q$ is a $\mathrm{DDO}$, ord $(Q)=m$ and $Q \geq 0$. Set

$$
L=\sum_{k=0}^{\infty} c^{-2 k} e^{-i k \theta} Q e^{i k \theta}
$$

Computing, we find that

$$
L-c^{-2} e^{-i \theta} L e^{i \theta}=Q \geq 0
$$

and $L$ is a Dirichlet operator with $\left\|M_{L}\right\| \leq c$. This establishes Proposition 3.11.

If $L$ is a Dirichlet operator, then $M_{L}$ is an operator on $H_{L}^{2}$ and thus $(y x-1)^{\ell}\left(M_{L}\right)$, as defined in Section 1, is an operator on $H_{L}^{2}$. The following proposition gives a formula for the sesquilinear form induced by this operator.

Proposition 3.13. If $L$ is a Dirichlet operator, $\ell \geq 0$, and $\varphi, \psi \in \mathcal{D}$, then

$$
\left[(y x-1)^{\ell}\left(M_{L}\right) \varphi, \psi\right]_{L}=\left[\left(\frac{d}{d D}\right)^{\ell} L\right](\varphi)(\bar{\psi})
$$

Proof.

$$
\begin{aligned}
{\left[(y x-1)^{\ell}\left(M_{L}\right) \varphi, \psi\right]_{L} } & =\left[\left(\sum_{k=0}^{\ell}(-1)^{\ell-k}\left(\begin{array}{l}
\ell \\
k
\end{array}\right) M_{L}^{* k} M_{L}^{k}\right) \varphi, \psi\right]_{L} \\
& =\sum_{k=0}^{\ell}(-1)^{\ell-k}\left(\begin{array}{l}
\ell \\
k
\end{array}\right)\left[e^{i k \theta} \varphi, e^{i k \theta} \psi\right]_{L} \\
& =\sum_{k=0}^{\ell}(-1)^{\ell-k}\left(\begin{array}{l}
\ell \\
k
\end{array}\right) e^{-i k \theta} L e^{i k \theta}(\varphi)(\bar{\psi}) \\
& =\left(\left(\frac{d}{d D}\right)^{\ell} L\right)(\varphi)(\bar{\psi}) \quad \text { (Lemma 2.4 iterated } \ell \text { times) }
\end{aligned}
$$

This establishes Proposition 3.13.

We finally are able to state and prove the first model theorem of this section. 
Theorem 3.14. If $\mathcal{H}$ is a Hilbert space, $m \geq 1, T \in \mathcal{L}(\mathcal{H})$ is an invertible $m$-isometry, and $\gamma \in \mathcal{H}$ is a bicyclic vector for $T$, then there exists a unique Dirichlet operator $L$ with ord $(L) \leq m-1$ and a Hilbert space isomorphism $U: \mathcal{H} \rightarrow H_{L}^{2}$ such that

$$
T=U^{*} M_{L} U
$$

and

$$
U(\gamma)=1
$$

Conversely, if $L$ is a Dirichlet operator with $\operatorname{ord}(L)=m-1$, then $M_{L}$ is an invertible $m$-isometry with bicyclic vector 1 .

Proof. First note that if $L$ is a Dirichlet operator, then by the definition of $H_{L}^{2}, 1$ is a bicyclic vector for $M_{L}$ (convergence in $\mathcal{D}$ implies convergence in $H_{L}^{2}$ ). Also note that if ord $(L)=m-1$, then

$$
\left(\frac{d}{d D}\right)^{m} L=0,
$$

a fact which implies via Proposition 3.13 that $M_{L}$ is an $m$-isometry. Since $\mathcal{D} \subseteq$ ran $M_{L}$, ran $M_{L}$ is dense and hence $M_{L}$ is invertible by Lemma 1.21 .

Conversely, let $\mathcal{H}, T$, and $\gamma$ be as in the statement of the theorem. Since $T$ is an $m$-isometry, the infinite bilateral array $\left\{a_{k_{1}, k_{2}}\right\}$ defined by

$$
a_{k_{1}, k_{2}}=\left\langle T^{k_{1}} \gamma, T^{k_{2}} \gamma\right\rangle
$$

is temperate and polynomial on diagonals of degree $\leq m-1$. Hence, by Proposition 2.7, there exists a DDO $L$ with ord $(L) \leq m-1$ such that

$$
\left\langle T^{k_{1}} \gamma, T^{k_{2}} \gamma\right\rangle=L\left(e^{i k_{1} \theta}\right)\left(e^{-i k_{2} \theta}\right)
$$

for all integers $k_{1}, k_{2}$.

By taking linear combinations of (3.15) and using the continuity of $L$ and Proposition 1.20 we deduce that

$$
\langle\varphi(T) \gamma, \psi(T) \gamma\rangle=L(\varphi)(\bar{\psi})
$$

for all $\varphi, \psi \in \mathcal{D}$

We first show that $L$ is a Dirichlet operator. If ord $(L)=0$, then (3.16) implies $L \geq 0$ and hence by the definition $L$ is a Dirichlet operator. If ord $(L) \geq 1$ observe that (3.16) implies that if $\varphi \in \mathcal{D}$, then

$$
\begin{aligned}
\left(L-c^{-2} e^{-i \theta} L e^{i \theta}\right)(\varphi)(\bar{\varphi})= & \left\langle\left(1-c^{-2} T^{*} T\right) \varphi(T) \gamma, \psi(T) \gamma\right\rangle \\
& \geq 0
\end{aligned}
$$


whenever $c \geq\|T\|$ (note: since ord $(L) \geq 1, T$ is not an isometry; thus $\|T\|>1$ ), and again $L$ is seen to be a Dirichlet operator.

Now, since $L$ is a Dirichlet operator, $H_{L}^{2}$ and $M_{L}$ are well-defined by the remark preceding Definition 3.10. To construct $U: \mathcal{H} \rightarrow H_{L}^{2}$, densely define an operator $U_{0}$ on $\mathcal{H}$ by the formula

$$
U_{0}(\varphi(T) \gamma)=\varphi \in H_{L}^{2}
$$

Observe that (3.16) implies that $U_{0}$ is well-defined and extends by continuity to a Hilbert space isomorphism $U: \mathcal{H} \rightarrow H_{L}^{2}$ ( $U_{0}$ is in fact defined densely, since $\gamma$ is bicyclic for $T$ and $U$ is surjective since $\mathcal{D} / M_{L}$ is dense in $H_{L}^{2}$ ).

Finally, note that by definition $U(\gamma)=1$ and that (3.17) implies $U T=M_{L} U$. This concludes the proof of Theorem 3.14.

If $\mathcal{H}$ is a Hilbert space, $T \in \mathcal{L}(\mathcal{H})$ is an invertible $m$-isometry and $\gamma \in \mathcal{H}$ is a nonzero vector, then $\gamma$ is bi-cyclic for $T \mid \bigvee\left\{T^{k} \gamma: k \in \mathbb{Z}\right\}$ and so there exists a uniquely associated Dirichlet operator given by Theorem 3.14. We will denote this associated Dirichlet operator by $(T, \gamma)^{\wedge}$.

The remainder of this section is devoted to three tasks. Firstly, we shall list without proof the rather straightforward analogs of Lemma 3.7, Definition 3.10, Proposition 3.11 and Proposition 3.13 for DTO, and Theorem 3.14 for the case of $m$-isometries not assumed to be invertible but possessing a cyclic vector. We then continue on to formulate notation that allows one to model multi-cyclic and multi-bicyclic $m$-isometries. This involves little more than allowing the coefficients in DDO and DTO to be matrices and the form spaces associated with them to be completions of $\mathcal{D}^{n}$ and $\mathcal{D}_{a}^{n}$, respectively. Finally. we close the section with a change of cyclic vector formula which turns out to have great value in the application of the model theory to the operator theory of $m$-isometries.

Lemma 3.18. Let $A$ be a DTO and fix $c>1 . A \geq 0$ and $M_{A}$ is a well-defined bounded operator on $H_{A}^{2}$ of norm $\leq c$ if and only if

$$
0 \leq A-c^{-2} e^{-i \theta} A e^{i \theta} .
$$

The following definition precisely identifies those DTO $A$ with the property that $A \geq 0$ and $M_{A}$ is a well-defined bounded operator.

Definition 3.20. An analytic Dirichlet operator is a DTO $A$ with the property that either there exists a constant $c>1$ such that

$$
0 \leq A-c^{-2} e^{-i \theta} A e^{i \theta},
$$

or ord $(A)=0$ and $A \geq 0$. 
Proposition 3.21. The analytic Dirichlet operators of order 0 consist of $P L \mid \mathcal{D}_{a}$ where $L$ is a positive measure. If $m \geq 1$, the analytic Dirichlet operators with ord $(A)=m$ and $\left\|M_{A}\right\| \leq c$ consist precisely of the set

$$
\left\{\sum_{k=0}^{\infty} c^{-2 k} e^{-i k \theta} Q e^{i k \theta}: Q \quad \text { is a } \quad D T O \quad \text { ord }(Q)=m, Q \geq 0\right\}
$$

Proposition 3.22. If $A$ is an analytic Dirichlet operator, $\ell \geq 0$, and $\varphi, \psi \in \mathcal{D}_{a}$, then

$$
\left[(y x-1)^{\ell}\left(M_{A}\right) \varphi, \psi\right]_{A}=\left(\frac{d}{d D}\right)^{\ell} A(\varphi)(\bar{\psi})
$$

Theorem 3.23. If $\mathcal{H}$ is a Hilbert space, $m \geq 1, T \in \mathcal{L}(\mathcal{H})$ is a $m$-isometry and $\gamma \in \mathcal{H}$ is a cyclic vector for $T$, then there exists a unique analytic Dirichlet operator $A$ with $\operatorname{ord}(A) \leq m-1$ and a Hilbert space isomorphism $U: \mathcal{H} \rightarrow H_{A}^{2}$ such that

$$
T=U^{*} M_{A} U
$$

and

$$
U(\gamma)=1
$$

Conversely, if $A$ is an analytic Dirichlet operator with ord $(A)=m-1$, then $M_{A}$ is an $m$-isometry with cyclic vector 1 .

If $T \in \mathcal{L}(\mathcal{H})$ is an $m$-isometry and $\gamma \in \mathcal{H}$ one can set

$$
\mathcal{H}_{\gamma}=\bigvee\left\{T^{k} \gamma: k \geq 0\right\}
$$

and

$$
T_{\gamma}=T \mid \mathcal{H}_{\gamma}
$$

With this setup, $T_{\gamma}$ is a cyclic $m$-isometry and $\gamma$ is a cyclic vector for $T_{\gamma}$. Accordingly by Theorem 3.23, there exists a unique analytic Dirichlet operator $A$ with the property that $\left(T_{\gamma}, \gamma\right)$ is unitarily equivalent to $\left(M_{A}, 1\right)$. We shall let $(T, \gamma)^{n}$ denote the unique DDO such that $A=P(T, \gamma) \hat{\mid} \mid \mathcal{D}_{a}$.

There is a certain amount of ambiguity in the $(T, \gamma)^{\wedge}$ notation. Observe that if $T \in$ $\mathcal{L}(\mathcal{H})$ is an invertible $m$-isometry and $\gamma \in \mathcal{H}$ then according to the remark following the proof of Theorem $3.14(T, \gamma)^{\wedge}$ is the unique Dirichlet operator such that

$$
\langle\varphi(T) \gamma, \psi(T) \gamma\rangle=(T, \gamma) \hat{(}(\varphi)(\bar{\psi})
$$


for all $\varphi, \psi \in \mathcal{D}$. On the other hand, $(T, \gamma)^{\wedge}$ as just defined in previous paragraph has the defining property

$$
\begin{aligned}
& \langle\varphi(T) \gamma, \psi(T) \gamma\rangle=A(\varphi)(\bar{\psi}) \\
& =(T, \gamma) \hat{(\varphi)}(\vec{\psi})
\end{aligned}
$$

for all $\varphi, \psi \in \mathcal{D}_{a}$. Recalling the remark following the proof of Lemma 3.3, we find that these two definitions of $(T, \gamma)^{\wedge}$ agree. The operator theory content of this observation is recorded without proof in the following proposition. If $T \in \mathcal{L}(\mathcal{H})$ is invertible and $\mathcal{M}$ is a subspace of $\mathcal{H}$, let us agree to say that $\mathcal{M}$ is bicyclic for $T$ if the smallest subspace of $\mathcal{H}$ containing $\mathcal{M}$ and invariant for $T$ and $T^{-1}$ is $\mathcal{H}$.

Proposition 3.24. Let $T_{1} \in \mathcal{L}\left(\mathcal{H}_{1}\right)$ and $T_{2} \in \mathcal{L}\left(\mathcal{H}_{2}\right)$ be invertible m-isometries. Let $\mathcal{M}_{1} \subseteq \mathcal{H}_{1}$ and $\mathcal{M}_{2} \subseteq \mathcal{H}_{2}$ be bicyclic invariant subspaces for $T_{1}$ and $T_{2}$ respectively. If $U: \mathcal{M}_{1} \rightarrow \mathcal{M}_{2}$ is a Hilbert space isomorphism such that $U T_{1}=T_{2} U$, then there exists a Hilbert space isomorphism $\tilde{U}: \mathcal{H}_{1} \rightarrow \mathcal{H}_{2}$ such that $\tilde{U} T_{1}=T_{2} \tilde{U}$ and $U=\tilde{U} \mid \mathcal{M}_{1}$.

Before continuing we explore some of the implications of Theorem 3.23 for cyclic 2 -isometries. Our first observation is that Proposition 1.23 implies that there are no nonunitary invertible 2-isometries. This implies via Theorem 3.14 that there are no Dirichlet operators of order 1 . Accordingly, the models for 2 -isometries are given only by analytic: Dirichlet operators.

Now, if $T \in \mathcal{L}(\mathcal{H})$ is a 2 -isometry and $\gamma \in \mathcal{H}$, then since the unilateral array $\left\langle T^{k_{1}} \gamma, T^{k_{2}} \gamma\right\rangle$ is linear on diagonals it is natural to define the slope $\mu$ and intercept $\beta$ of $(T, \gamma)$ to be the elements of $\mathcal{D}^{\prime}$ defined by the formulas

$$
\hat{\mu}(k)=\left\{\begin{array}{cc}
\left\langle T^{k+1} \gamma, T \gamma\right\rangle-\left\langle T^{k} \gamma, \gamma\right\rangle & k \geq 0 \\
\left\langle T \gamma, T^{-k+1} \gamma\right\rangle-\left\langle\gamma, T^{-k} \gamma\right\rangle & k<0
\end{array}\right.
$$

and

$$
\hat{\beta}(k)=\left\{\begin{array}{cc}
\left\langle T^{k} \gamma, \gamma\right\rangle & k \geq 0 \\
\left\langle\gamma, T^{-k} \gamma\right\rangle & k<0
\end{array}\right.
$$

On the other hand there exist a pair of distributions $\beta_{0}$ and $\beta_{1}$ such that

$$
A=P\left(\beta_{1} D+\beta_{0}\right) \mid \mathcal{D}_{a}
$$

Furthermore, the formulas that relate the above distributions are given by

$$
\beta_{1}=\mu ; \quad \text { and }
$$

$$
\beta_{0}=\beta+(1-P)(D \mu)
$$


Now if one is given a 2-isometry $T \in \mathcal{L}(\mathcal{H})$, then by varying the choice of vector $\gamma$ one can obtain various slopes and intercepts. Three choices have been considered. In [R2] Richter chooses $\gamma \in \operatorname{ker} T^{*}$. Evidently,via (3.26) one sees that this corresponds to requiring the intercept of $(T, \gamma)$ to be a scalar multiple of $\frac{d}{d \theta}$. In Section 6 we shall analyze the case where $\gamma$ is chosen so that the slope of $(T, \gamma)$ is $\frac{d}{d \theta}$. In Section 8 we shall investigate the case where the slope and intercept are chosen colinear.

Before continuing we record the following simple reformulation of Lemma 3.18 in the case where ord $(A)=1$.

Lemma 3.28. Let $A=P\left(\beta_{1} D+\beta_{0}\right) \mid \mathcal{D}_{a}$ be a DTO. $A$ is an analytic Dirichlet operator if and only if there exists $\varepsilon>0$ such that

$$
\varepsilon P \beta_{1} \mid \mathcal{D}_{a} \leq A .
$$

Furthermore, if the covariance of $A(\operatorname{cov}(A))$ is defined by

$$
\operatorname{cov}(A)=\left\|\triangle_{M_{A}}\right\|^{\frac{1}{2}}
$$

then

$$
\operatorname{cov}(A)^{2}=\min \left\{\frac{1}{\varepsilon}: \varepsilon \quad \text { satisfies }(3.29)\right\}
$$

We now turn to the problem of modeling multicyclic $m$-isometries. We shall do the case of invertible $m$-isometries in some detail and merely state the notation and results for the noninvertible case.

If $n_{1}$ and $n_{2}$ are positive integers, recall that $\mathcal{D}^{n_{1}, n_{2}}$ denotes the space of $n_{1} \times n_{2}$ matrices with entries in $\mathcal{D}$. Likewise, let $\mathrm{DDO}_{m}^{n_{1}, n_{2}}$ denote the space of $n_{1} \times n_{2}$ matrices with DDO entries $L_{r s}$ with the order of $L_{r s} \leq m$.

$$
\begin{gathered}
\text { If } \psi=\left(\begin{array}{c}
\psi_{1} \\
\vdots \\
\psi_{n}
\end{array}\right) \in \mathcal{D}^{n, 1} \text {, we define } \psi^{*} \in \mathcal{D}^{1, n} \text { by setting } \\
\psi^{*}=\left(\bar{\psi}_{1}, \ldots, \bar{\psi}_{n}\right) .
\end{gathered}
$$

Our first observation is that $\left(\mathcal{D}^{1, n}\right)^{\prime}$ can be identified with $\left(\mathcal{D}^{\prime}\right)^{n, 1}$ via the pairing

$$
u(\psi)=\sum_{r=1}^{n} u_{r}\left(\psi_{r}\right)
$$

where $\psi=\left(\psi_{r}\right) \in \mathcal{D}^{1, n}$ and $u=\left(u_{r}\right) \in\left(\mathcal{D}^{\prime}\right)^{n, 1}$. Note also, that if $L=\left(L_{r s}\right) \in \mathrm{DDO}_{m}^{n, n}$ then $L$ induces a linear mapping from $\mathcal{D}^{n, 1}$ into $\left(\mathcal{D}^{\prime}\right)^{n, 1}$ via the formula

$$
L(\varphi)=\left(\sum_{s} L_{r s}\left(\varphi_{s}\right)\right)
$$


Combining (3.30) and (3.31) leads to the formula

$$
L(\varphi)\left(\psi^{*}\right)=\sum_{r s} L_{r s}\left(\varphi_{s}\right)\left(\bar{\psi}_{r}\right)
$$

for all $\varphi, \psi \in \mathcal{D}^{n, 1}$.

We now point out that with the notations of the preceding paragraph that all of the results about scalar DDO of Section 2 have analogs for the case of matrix DDO. In particular, note that if $L \in \mathrm{DDO}_{m}^{n, n}$, then there exist unique $\beta_{\ell} \in\left(\mathcal{D}^{\prime}\right)^{n, n}$ with

$$
L=\sum_{\ell=0}^{m} \beta_{\ell} D^{(\ell)}
$$

and if $\frac{d}{d D} L$ is defined as in Definition 2.3 by setting

$$
\frac{d}{d D} L=\sum \ell \beta_{\ell} D^{(\ell-1)}
$$

then the following three formulas hold for all $\varphi, \psi \in \mathcal{D}^{n, 1}$.

$$
\begin{gathered}
e^{-i \theta} L e^{i \theta}-L=\frac{d}{d D} L . \\
\left.\delta^{m}\left\{L\left(e^{i k \theta} \varphi\right)\left(e^{-i k \theta} \psi^{*}\right)\right\}\right|_{k=0}=\left(\frac{d}{d D}\right)^{m} L(\varphi)\left(\psi^{*}\right) \\
\lim _{k \rightarrow \infty} \frac{1}{k^{(m)}} e^{-i k \theta} L e^{i k \theta}(\varphi)\left(\psi^{*}\right)=\beta_{m}\left(\varphi \psi^{*}\right) .
\end{gathered}
$$

Furthermore, the formula

$$
\left\langle a_{k_{1}, k_{2}} u, v\right\rangle=L\left(e^{i k_{1} \theta} u\right)\left(e^{-i k_{2} \theta} v^{*}\right), \quad u, v \in \mathbb{C}^{n, 1}
$$

sets up a $1-1$ correspondence between $n \times n$ matrix DDO $L$ of order $m$ and bilateral (resp.. unilateral) temperate $n \times n$ block matrix arrays which are polynomial on diagonals of degree $m$ as in Proposition 2.7 (resp. Cor. 2.10).

Finally we record the following generalization of Proposition 2.14. Note that if $L$ : $\mathcal{D}^{n, 1} \rightarrow\left(\mathcal{D}^{\prime}\right)^{n, 1}$ is a continuous linear mapping, then a matrix distribution can be defined via the Schwarz nuclear theorem by requiring

$$
u\left(\varphi\left(e^{i \theta_{1}}\right) \psi\left(e^{i \theta_{2}}\right)^{*}\right)=L(\varphi)\left(\psi^{*}\right), \quad \varphi, \psi \in \mathcal{D}^{n, 1}
$$


Proposition 3.37. The following conditions on a continuous linear mapping $L: \mathcal{D}^{n, 1} \rightarrow$ $\left(\mathcal{D}^{\prime}\right)^{n, 1}$ are equivalent.

(i) There exists an $m \geq 0$ such that

$$
\sum_{\ell=0}^{m}(-1)^{m-\ell}\left(\begin{array}{c}
m \\
\ell
\end{array}\right) e^{-i \ell \theta} L e^{i \ell \theta}=0
$$

(ii) $L$ is a $D D O$.

(iii) The distribution $u$ defined by (3.36) has support in the set

$$
\left\{\left(e^{i \theta}, e^{i \theta}\right): \theta \in \mathbb{R}\right\} \text {. }
$$

If $L \in \mathrm{DDO}_{m}^{n, n}$, let us agree to say that $L$ is positive $(L \geq 0)$ if $L(\varphi)\left(\varphi^{*}\right) \geq 0$ whenever $\varphi \in \mathcal{D}^{n, 1}$.

If $L \in \mathrm{DDO}_{m}^{n, n}$, we define the form space of $L, H_{L}^{2}$, to be the completion of $\mathcal{D}^{n, 1}$ with respect to the sesquilinear form $[\cdot, \cdot]_{L}$ on $\mathcal{D}^{n, 1} \times \mathcal{D}^{n, 1}$ given by

$$
[\varphi, \psi]_{L}=L(\varphi)\left(\psi^{*}\right) \text {. }
$$

The following lemma describes when multiplication by $e^{i \theta}$ on $H_{L}^{2}, M_{L}$, is well-defined and bounded. Its proof is identical to that of Lemma 3.7.

Lemma 3.38. Let $L \in D D O_{m}^{n, n}$ and fix $c>1 . L \geq 0$ and $M_{L}$ is a well-defined bounded operator on $H_{L}^{2}$ of norm $\leq c$ if and only if

$$
0 \leq L-c^{-2} e^{-i \theta} L e^{i \theta}
$$

As in the case of scalar DDO, we say that $L \in \mathrm{DDO}_{m}^{n, n}$ is a Dirichlet operator if either there exists a constant $c>1$ such that (3.39) holds or ord $(L)=0$ and $L \geq 0$. It is then the case that $L \in \mathrm{DDO}_{m}^{n, n}$ is a Dirichlet operator if and only if $L \geq 0$ and $M_{L}$ is bounded.

If $c>1$ is a given constant, then the Dirichlet operators $L \in \mathrm{DDO}_{m}^{n, n}$ with $\left\|M_{L}\right\| \leq$ $c$ can be completely parameterized via an affine transformation by positive $\mathrm{DDO}_{m}^{n, n}$ as described in the following proposition. The proof is identical to that of Proposition 3.11.

Proposition 3.40. If $m \geq 1$, the Dirichlet operators $L \in D D O_{m}^{n, n}$ with $\left\|M_{L}\right\| \leq c$ consist precisely of the set

$$
\left\{\sum_{k=0}^{\infty} c^{-2 k} e^{-i k \theta} Q e^{i k \theta}: Q \in D D O_{m}^{n, n}, Q \geq 0\right\} .
$$

If $L \in \mathrm{DDO}_{m}^{n, n}$ is a Dirichlet operator, then $M_{L}$ is an operator on $H_{L}^{2}$ and thus $(y x-1)^{\ell}\left(M_{L}\right)$, as defined in Section 1 , is an operator on $H_{L}^{2}$. The following proposition gives a formula for the sesquilinear form induced by this operator. It follows from (3.33) in exactly the same way that Proposition 3.13 followed from Lemma 2.4. 
Proposition 3.41. If $L \in D D O_{m}^{n, n}$ is a Dirichlet operator, $\ell \geq 0$ and $\varphi, \psi \in \mathcal{D}^{n, 1}$, then

$$
\left[(y x-1)^{\ell}\left(M_{L}\right) \varphi, \psi\right]_{L}=\left[\left(\frac{d}{d D}\right)^{\ell} L\right](\varphi)\left(\psi^{*}\right)
$$

For $1 \leq r \leq n$, we define $e_{r} \in \mathcal{D}^{n, 1}$ to be the vector whose $r$ th coordinate is $1 \in \mathcal{D}$ and whose other coordinates are 0 .

We finally are able to state and prove the model theorem for finitely bicyclic invertible $m$-isometries. For $T \in \mathcal{L}(\mathcal{H}), \gamma=\left(\gamma_{r}\right) \in \mathcal{H}^{n, 1}$, and $p$ a $1 \times n$ row vector of polynomials let us agree to define $p(T) \in \mathcal{L}(\mathcal{H})^{1, n}$ by

$$
p(T)=\left(p_{r}(T)\right)
$$

Since $\mathcal{L}(\mathcal{H})^{1, n}$ acts naturally on $\mathcal{H}^{n, 1}$ this leads to the notation,

$$
p(T) \gamma=\sum p_{r}(T) \gamma_{r}
$$

In particular if $T$ is an invertible $m$-isometry, then by Proposition 1.20, $T$ possesses a $\mathcal{D}$-functional calculus and by continuity $(3.42)$ becomes

$$
\varphi(T) \gamma=\sum \varphi_{r}(T) \gamma_{r} \quad, \quad \varphi \in \mathcal{D}^{1, n}
$$

Theorem 3.44. If $\mathcal{H}$ is a Hilbert space, $m \geq 1, T \in \mathcal{L}(\mathcal{H})$ is an invertible $m$-isometry, and $\gamma=\left(\gamma_{r}\right) \in \mathcal{H}^{n, 1}$ is an $n$-bicyclic tuple for $T$, then there exist a unique Dirichlet operator $L \in D D O_{m-1}^{n, n}$ and a Hilbert space isomorphism $U: \mathcal{H} \rightarrow H_{L}^{2}$ such that

$$
T=U^{*} M_{L} U
$$

and

$$
U\left(\gamma_{r}\right)=e_{r} \quad 1 \leq r \leq n
$$

Conversely, if $L \in D D O_{m-1}^{n, n}$ is a Dirichlet operator, then $M_{L}$ is an invertible $m$-isometry with bicyclic $n$-tuple $\left(e_{r}\right)$.

ProOF. First note that if $L \in \mathrm{DDO}_{m-1}^{n, n}$ is a Dirichlet operator, then by the definition of $H_{L}^{2}, e_{1}, \ldots, e_{n}$ are bicyclic vectors for $M_{L}$ (convergence in $\mathcal{D}^{1, n}$ implies convergence in $H_{L}^{2}$ ). Also note that

$$
\left(\frac{d}{d D}\right)^{m} L=0
$$


a fact which implies via Proposition 3.41 that $M_{L}$ is an $m$-isometry. Since $\mathcal{D}^{n, 1} \subseteq$ ran $M_{L}$, ran $M_{L}$ is dense and hence $M_{L}$ is invertible by Lemma 1.21 .

Conversely, let $\mathcal{H}, T$, and $\gamma_{1}, \ldots, \gamma_{n}$ be as in the statement of the theorem. We construct $L \in \mathrm{DDO}_{m-1}^{n, n}$ componentwise. Accordingly, fix $r, s \in\{1, \ldots, n\}$. Since $T$ is an $m$-isometry, the infinite bilateral array $\left\{a_{k_{1}, k_{2}}\right\}$ defined by

$$
a_{k_{1}, k_{2}}=\left\langle T^{k_{1}} \gamma_{r}, T^{k_{2}} \gamma_{s}\right\rangle
$$

is temperate and polynomial on diagonals of degree $\leq m-1$. Hence by Proposition 2.7, there exists a DDO $L_{r s}$ with ord $(L) \leq m-1$ such that

$$
L_{r s}\left(e^{i k_{1} \theta}\right)\left(e^{-i k_{2} \theta}\right)=\left\langle T^{k_{1}} \gamma_{s}, T^{k_{2}} \gamma_{r}\right\rangle
$$

for all integers $k_{1}, k_{2}$.

By taking linear combinations of (3.45) and using the continuity of $L$ and Lemma 1.20 we deduce that

$$
L_{r s}(\varphi)(\bar{\psi})=\left\langle\varphi(T) \gamma_{s}, \psi(T) \gamma_{r}\right\rangle
$$

for all $\varphi, \psi \in \mathcal{D}$.

Set $L=\left(L_{r s}\right) \in \mathrm{DDO}_{m-1}^{n, n}$. If $\varphi, \psi \in \mathcal{D}^{n, 1}$, then

$$
\begin{aligned}
L(\varphi)\left(\psi^{*}\right) & =\sum_{r, s=1}^{n} L_{r s}\left(\varphi_{s}\right)\left(\bar{\psi}_{r}\right) \\
& =\sum_{r, s}\left\langle\varphi_{s}(T) \gamma_{s}, \psi_{r}(T) \gamma_{r}\right\rangle \\
& =\left\langle\sum_{s} \varphi_{s}(T) \gamma_{s}, \sum_{r} \psi_{r}(T) \gamma_{r}\right\rangle \\
& =\left\langle\varphi^{\tau}(T) \gamma, \psi^{\tau}(T) \gamma\right\rangle
\end{aligned}
$$

a fact which we record as

$$
L(\varphi)\left(\psi^{*}\right)=\left\langle\varphi^{\tau}(T) \gamma, \psi^{\tau}(T) \gamma\right\rangle
$$

If ord $(L)=0$, then (3.47) implies $L \geq 0$ and hence by definition $L$ is a Dirichlet operator. To see that $L$ is a Dirichlet operator when ord $(L) \geq 1$ observe that (3.47) implies that if $\varphi \in \mathcal{D}^{n, 1}$ and $c=\|T\|$, then

$$
\begin{aligned}
\left(L-c^{-2} e^{-i \theta} L e^{i \theta}\right)(\varphi)\left(\varphi^{*}\right)= & \left\langle\left(1-c^{-2} T^{*} T\right) \varphi(T) \gamma, \varphi^{T}(T) \gamma\right\rangle \\
& \geq 0
\end{aligned}
$$


(note: since ord $(L) \geq 1, T$ is not an isometry; thus $\|T\|>1$ ). Thus, $L$ is a Dirichlet operator and both $H_{L}^{2}$ and $M_{L}$ are well-defined.

To construct $U: \mathcal{H} \rightarrow H_{L}^{2}$, densely define an operator $U_{0}$ on $\mathcal{H}$ by the formula

$$
U_{0}\left(\sum_{r=1}^{n} \varphi_{r}(T) \gamma_{r}\right)=\left(\varphi_{r}\right) \in H_{L}^{2}
$$

Observe that (3.47) implies that $U_{0}$ is well-defined and extends by continuity to a Hilbert space isomorphism $U: \mathcal{H} \rightarrow H_{L}^{2}, U_{0}$ is in fact defined densely, since $\gamma_{1}, \ldots, \gamma_{n}$ are bicyclic for $T$ and $U$ is surjective since $\mathcal{D}^{n, 1} /\left\{\varphi \in \mathcal{D}^{n, 1}: L(\varphi)\left(\varphi^{*}\right)=0\right\}$ is dense in $H_{L}^{2}$.

Finally note that by definition $U\left(\gamma_{j}\right)=e_{j}$ and that (3.48) implies $U T=M_{L} U$. This concludes the proof of Theorem 3.44 .

If $\mathcal{H}$ is a Hilbert space, $T \in \mathcal{L}(\mathcal{H})$ is an invertible $m$-isometry and $\gamma_{1}, \ldots, \gamma_{n} \in \mathcal{H}$, then $\gamma=\left(\gamma_{1}, \ldots, \gamma_{n}\right)$ is a bicyclic $n$-tuple for

$$
T \mid \bigvee\left\{T^{k} \gamma_{j}: k \in \mathbb{Z} \quad 1 \leq j \leq n\right\}
$$

and so there exists a uniquely associated matricial Dirichlet operator given by Theorem 3.44. We will denote this associated matricial Dirichlet operator by $(T, \gamma)$.

Letting $\mathrm{DTO}_{m}^{n_{1}, n_{2}}$ denote the space of $n_{1} \times n_{2}$ matrices with DTO entries $A_{r s}$ with the order of $A_{r s} \leq m$, we can then define

$$
A(\varphi)\left(\psi^{*}\right) \quad \text { for } \varphi, \psi \in \mathcal{D}_{a}^{n, 1},
$$

positivity of $A$, the form space of $A, H_{A}^{2}, M_{A}$, multiplication by $e^{i \theta}$ on $H_{A}^{2}$ and matricial analytic Dirichlet operator in the obvious way. The straightforward analogs of Lemma 3.38, Proposition 3.40, Proposition 3.41 hold and lead to the following model theorem for multicyclic $m$-isometries not assumed to be invertible.

Theorem 3.49. If $\mathcal{H}$ is a Hilbert space, $m \geq 1, T \in \mathcal{L}(\mathcal{H})$ is an m-isometry and $\gamma=$ $\left(\gamma_{r}\right) \in \mathcal{H}^{n, 1}$, is a $n$-cyclic tuple for $T$, then there exists a unique analytic Dirichlet operator $A \in D T O_{m-1}^{n, n}$ and a Hilbert space isomorphism $U: \mathcal{H} \rightarrow H_{A}^{2}$ such that

$$
T=U^{*} M_{A} U
$$

and

$$
U\left(\gamma_{r}\right)=e_{r} \quad 1 \leq r \leq n .
$$

Conversely, if $A \in D T O_{m-1}^{n, n}$ is an analytic Dirichlet operator, then $M_{A}$ is an m-isometry with cyclic $n$-tuple $\left(e_{r}\right)$.

Now, as in the case of invertible $m$-isometries, we introduce the following notation. If $T \in \mathcal{L}(\mathcal{H})$ is an $m$-isometry, $\gamma=\left(\gamma_{1}, \ldots, \gamma_{n}\right) \in \mathcal{H}^{n, 1}$ is a choice of $n$ vectors and $\mathcal{H}_{\gamma} \subseteq \mathcal{H}$ is defined by

$$
\mathcal{H}_{\gamma}=\bigvee\left\{T^{k} \gamma_{r}: k \geq 0, \quad 1 \leq r \leq n\right\}
$$


then $T \mid \mathcal{H}_{\gamma}$ is an $m$-isometry and $\gamma$ is a cyclic $n$-tuple for $T \mid \mathcal{H}_{\gamma}$. Accordingly, by Theorem 3.49 there exists a unique $A \in \mathrm{DTO}_{m-1}^{n, n}$ with the stated properties of that theorem. In the sequel we shall define $(T, \gamma)^{\wedge}$ to be the unique DDO such that $A=P(T, \gamma)^{\wedge} \mid \mathcal{D}_{a}^{n, 1}$.

Now, suppose one is presented with a finitely cyclic $m$-isometry $T$ and wishes to use Theorem 3.49 to model $T$. Since in general $T$ will have many cyclic $n$-tuples, there are many associated DTO $A$. This phenomenon is already present in the case of isometries. To be precise, let $T$ be a cyclic isometry. Thus, $T$ is unitarily equivalent to $M_{e^{i \theta} \theta}$ on $H^{2}(\mu)$ for some positive measure $\mu$ supported on the circle. In this case a well-known piece of function theory gives that

$$
H^{2}(\mu)=L^{2}\left(\mu_{s}\right) \oplus H^{2}\left(w \frac{d \theta}{2 \pi}\right)
$$

where $d \mu=d \mu_{s}+w \frac{d \theta}{2 \pi}$ is the Lebesgue decomposition of $\mu$. Furthermore,

$$
H^{2}\left(w \frac{d \theta}{2 \pi}\right)=\left\{\begin{array}{cc}
\frac{1}{f} H^{2} & \text { if } \int \ln w \frac{d \theta}{2 \pi}>-\infty \\
L^{2}\left(w \frac{d \theta}{2 \pi}\right) & \text { if } \int \ln w \frac{d \theta}{2 \pi}=-\infty
\end{array}\right.
$$

where $f \in H^{2}$ is the outer function such that $|f|^{2}=w$. Assume that $T$ is not unitary so that $\int \ln w \frac{d \theta}{2 \pi}>-\infty$. With this model, a vector $\gamma=\varphi \oplus g$ is cyclic for $T$ if and only if $f g$ is outer and $\varphi \neq 0$ for $\mu_{s}$ a.e. $e^{i \theta}$. Furthermore, it is easy to check that

$$
(T, \gamma)^{\wedge}=|\varphi|^{2} d \mu_{s}+|g f|^{2} \frac{d \theta}{2 \pi} .
$$

Of course, in the theory of isometries, one knows in advance via the wandering subspace argument that $\gamma$ can be chosen so that $g f=1$ in (3.50), so that the pure cyclic isometry is unique and unitarily equivalent to $M_{e^{i \theta}}$ on $H^{2}$. When $m \geq 1$, however, there is no preordained choice for $\gamma$ (recall the remarks made in the paragraph following (3.27)) and analogs of formula (3.50) have added interest. The following theorem shows how $(T, \gamma)^{\wedge}$ transforms when $\gamma$ is transformed via the functional calculus.

For $F=\left(F_{r s}\right) \in \mathcal{D}^{n_{2}, n_{1}}$ define $F^{\tau}$ and $F^{*}$ by

$$
\begin{aligned}
& F^{\tau}=\left(F_{s r}\right), \quad \text { and } \\
& F^{*}=\left(\bar{F}_{s r}\right) .
\end{aligned}
$$

Now if $F \in \mathcal{D}^{n_{2} n_{1}}$ then $F$ induces a map $F: \mathcal{D}^{n_{1}, 1} \rightarrow \mathcal{D}^{n_{2}, 1}$ so that if $L \in \mathrm{DDO}_{m}^{n_{3}, n_{2}}$ then $L F: \mathcal{D}^{n_{1}, 1} \rightarrow\left(\mathcal{D}^{\prime}\right)^{n_{3}, 1}$. Concretely,

$$
L F=\left(\sum_{t} L_{r t} F_{t s}\right) .
$$


Similarly, if $G \in \mathcal{D}^{n_{4}, n_{3}}$, then $G$ induces a map $G: \mathcal{D}^{n_{3}, 1} \rightarrow \mathcal{D}^{n_{4}, 1}$ so that $G L$ represents a map $G L: \mathcal{D}^{n_{2}, 1} \rightarrow\left(\mathcal{D}^{\prime}\right)^{n_{4}, 1}$.

Now recall that if $T$ is an $m$-isometry, $\gamma \in \mathcal{H}^{n_{1}, 1}$, and $\varphi \in \mathcal{D}_{a}^{1, n_{1}}$, then $\varphi(T) \gamma \in \mathcal{H}$ is defined by

$$
\varphi(T) \gamma=\sum_{s} \varphi_{s}(T) \gamma_{s}
$$

More generally, if $F \in \mathcal{D}_{a}^{n_{2}, n_{1}}$ define $F(T) \gamma \in \mathcal{H}^{n_{2}, 1}$ by

$$
F(T) \gamma=\left(\sum_{s} F_{r s} \gamma_{s}\right)
$$

Theorem 3.52. Fix positive integers $m, n_{1}$, and $n_{2}$. If $T$ is an $m$-isometry, $\gamma \in \mathcal{H}^{n_{1}, 1}$, $F \in \mathcal{D}_{a}^{n_{2}, n_{1}}$ and $\ell \geq 0$, then

$$
\left(\frac{d}{d D}\right)^{\ell}(T, F(T) \gamma)^{\wedge}=F^{\tau *}\left(\left(\frac{d}{d D}\right)^{\ell}(T, \gamma)^{\wedge}\right) F^{\tau}
$$

Proof. Let $\mu=F(T) \gamma$ and set $L=(T, \gamma)^{\hat{y}}$. We first show that (3.53) holds when $\ell=0$. Thus we need to show that

$$
(T, \mu)_{k \ell}=\left(F^{r *} L F^{\top}\right)_{k \ell}
$$

for all $k, \ell \leq n_{2}$. But if $\varphi, \psi \in \mathcal{D}_{a}$, then

$$
\begin{aligned}
(T, \mu)_{k \ell}(\varphi)(\bar{\psi}) & =\left\langle\varphi(T) \mu_{\ell}, \psi(T) \mu_{k}\right\rangle \\
& =\left\langle\varphi(T) \sum_{r} F_{\ell r}(T) \gamma_{r}, \psi(T) \sum_{s} F_{k s}(T) \gamma_{s}\right\rangle \\
& =\sum_{r, s}\left\langle\left(\varphi F_{\ell r}\right)(T) \gamma_{r},\left(\psi F_{k s}\right)(T) \gamma_{s}\right\rangle \\
& =\sum_{r, s} L_{s r}\left(\varphi F_{\ell r}\right)\left(\overline{\psi F_{k s}}\right) \\
& =\sum_{r, s}\left(\overline{F_{k s}} L_{s r} F_{\ell r}\right)(\varphi)(\bar{\psi}) \\
& =\left(F^{\tau *} L F^{\tau}\right)_{k \ell}(\varphi)(\bar{\psi})
\end{aligned}
$$

To deduce that (3.53) holds when $\ell>0$ simply apply the identity

$$
\frac{d}{d D} G^{*} L G=G^{*}\left(\frac{d}{d D} L\right) G
$$

which holds for all $L \in \mathrm{DDO}_{m}^{n_{1}, n_{1}}$ and $G \in \mathcal{D}^{n_{1}, n_{2}}$. This establishes Theorem 3.52 .

\section{$\S 4$. Smoothing applied to $m$-isometries}

In this section, we describe how to convolve smooth functions with DDO, DTO and $m$-isometries $T$. This allows one to reduce many qualitative questions concerning the operator theory of $m$-isometries to the case where the $m$-isometry possesses a model based 
on a DDO with $C^{\infty}$ coefficients, i.e. a classical differential operator. A concrete example of this procedure will be given in Section 7 where we shall show using the Arveson Extension Theorem that the model theory for 2-isometries can be understood in terms of a Disconjugacy Theorem for the underlying DTO.

Recall that if $\varphi \in \mathcal{D}$ and $t \in \mathbb{R}$, then the translation of $\varphi$ by $t, \varphi_{t}$, is defined by $\varphi_{t}\left(e^{i \theta}\right)=\varphi\left(e^{i(\theta-t)}\right)$ for $\theta \in \mathbb{R}$. If $u \in \mathcal{D}^{\prime}$, then the translation of $u$ by $t \in \mathbb{R}, u_{t}$, is the distribution defined by $u_{t}(\varphi)=u\left(\varphi_{-t}\right)$. Finally, if $\omega \in \mathcal{D}$ and $u \in \mathcal{D}^{\prime}$, then the convolution of $\omega$ and $u, \omega * u$, is defined by the formula,

$$
(\omega * u)=\int \omega\left(e^{i t}\right) u_{t} \frac{d t}{2 \pi} .
$$

These notions can be extended to translations and convolutions of elements of $\mathcal{D}^{n_{1}, n_{2}}$ and $\left(\mathcal{D}^{\prime}\right)^{n_{1}, n_{2}}$ componentwise.

We now extend these notions to DDO.

Definition 4.1. If $L: \mathcal{D}^{n, 1} \rightarrow\left(\mathcal{D}^{\prime}\right)^{n, 1}$ is continuous and linear and $t \in \mathbb{R}$, then we define the translation of $L$ by $t, L_{t}: \mathcal{D}^{n, 1} \rightarrow\left(\mathcal{D}^{\prime}\right)^{n, 1}$, by the formula

$$
L_{t}(\varphi)=(L(\varphi-t))_{t} \text {. }
$$

Proposition 4.2. Fix $t \in \mathbb{R}$ and integers $m \geq 0, n \geq 1$. If

$$
L=\sum_{\ell=0}^{m} \beta_{\ell} D^{(\ell)} \in D D O_{m}^{n, n},
$$

then

$$
L_{t}=\sum_{\ell=0}^{m}\left(\beta_{\ell}\right)_{t} D^{(\ell)} .
$$

In addition, if $L$ is a Dirichlet operator, then $L_{t}$ is a Dirichlet operator with $\left\|M_{L_{t}}\right\|=\left\|M_{L}\right\|$ and if $A=P L \mid \mathcal{D}_{a}$ is an analytic Dirichlet operator, then $A_{t}=P L_{t} \mid \mathcal{D}_{a}$ is an analytic Dirichlet operator with $\left\|M_{A_{t}}\right\|=\left\|M_{A}\right\|$.

ProOF. First recall that $D$ commutes with translation. Computing, we find that if $\varphi \in$ $\mathcal{D}^{n, 1}$ and $\psi \in \mathcal{D}^{1, n}$

$$
\begin{aligned}
\left(\beta_{\ell} D^{(\ell)}\right)_{t}(\varphi)(\psi) & =\left(\beta_{\ell} D^{(\ell)}\left(\varphi_{-t}\right)\right)_{t}(\psi) \\
& =\beta_{\ell} D^{(\ell)}\left(\varphi_{-t}\right)\left(\psi_{-t}\right) \\
& =\beta_{\ell}\left(\left(D^{(\ell)}\left(\varphi_{-t}\right)\right) \psi_{-t}\right) \\
& =\beta_{\ell}\left(\left(D^{(\ell)} \varphi\right)_{-t} \psi_{-t}\right) \\
& =\left(\beta_{\ell}\right)_{t}\left(\left(D^{(\ell)} \varphi\right) \psi\right) \\
& =\left(\left(\beta_{\ell}\right)_{t} D^{(\ell)}\right)(\varphi)(\psi)
\end{aligned}
$$


Thus (4.3) holds.

Suppose now that $L$ is a Dirichlet operator. If ord $(L)=0$, then $L \geq 0$. Consequently ord $\left(L_{t}\right)=0$ and $L_{t} \geq 0$. Hence $L_{t}$ is a Dirichlet operator. On the other hand if ord $(L) \geq 1$, then by (3.39), there is a $c>1$ such that

$$
0 \leq L-e^{-2} e^{-i \theta} L e^{i \theta}
$$

If $\varphi \in \mathcal{D}^{n, 1}$, then

$$
\begin{aligned}
\left(L_{t}-c^{-2} e^{-i \theta} L_{t} e^{i \theta}\right)(\varphi)\left(\varphi^{*}\right)= & L\left(\varphi_{-t}\right)\left(\varphi_{-t}^{*}\right)-c^{-2} L_{t}\left(e^{i \theta} \varphi\right)\left(\left(e^{i \theta} \varphi\right)^{*}\right) \\
= & L\left(\varphi_{-t}\right)\left(\varphi_{-t}^{*}\right)-c^{-2} L\left(e^{i(\theta+t)} \varphi_{-t}\right)\left(\left(e^{i(\theta+t)} \varphi_{-t}\right)^{*}\right) \\
= & L\left(\varphi_{-t}\right)\left(\varphi_{-t}^{*}\right)-c^{-2}\left(e^{-i \theta} L e^{i \theta}\right)\left(\varphi_{-t}\right)\left(\varphi_{-t}^{*}\right) \\
& \geq 0 .
\end{aligned}
$$

Thus, $L_{t}$ is a Dirichlet operator. Furthermore since $\left\|M_{L}\right\|$ is the smallest $c$ such that (4.4) holds, this computation shows that in fact $\left\|M_{L}\right\|=\left\|M_{L_{t}}\right\|$.

The proof of Proposition 4.2 is now completed by doing an analogous argument with $L$ replaced by $P L \mid \mathcal{D}_{a}$.

Now, Proposition 4.2 implies that if $T \in \mathcal{L}(\mathcal{H})$ is an $m$-isometry, $\gamma \in \mathcal{H}^{n, 1}$ and $t \in \mathbb{R}$, then $((T, \gamma))_{t}$ is a $n \times n$ matricial Dirichlet operator and thus via Theorem 3.44 models some operator $T_{t}$.

Proposition 4.5. Let $T \in \mathcal{L}(\mathcal{H})$ be an $m$-isometry, $\gamma \in \mathcal{H}^{n, 1}$ and $t \in \mathbb{R}$. If $T_{t}=e^{i t} T$; $\left(T_{t}, \gamma\right)^{\wedge}=\left((T, \gamma)^{\wedge}\right)_{t}$

Proof. First note that $\varphi_{-t}(T)=\varphi\left(T_{t}\right)$. Thus, for $\varphi, \psi \in \mathcal{D}_{a}^{n, 1}$, we find that

$$
\begin{aligned}
(T, \gamma)_{t}(\varphi)\left(\psi^{*}\right) & =(T, \gamma) \hat{(}\left(\varphi_{-t}\right)\left(\psi_{-t}^{*}\right) \\
& =\left\langle\varphi_{-t}^{\tau}(T) \gamma, \psi_{-t}^{\tau}(T) \gamma\right\rangle \\
& =\left\langle\varphi^{\tau}\left(T_{t}\right) \gamma, \psi^{\tau}\left(T_{t}\right) \gamma\right\rangle \\
& =\left(T_{t}, \gamma\right)(\varphi)\left(\psi^{*}\right) .
\end{aligned}
$$

This establishes Proposition 4.5.

Definition 4.6 If $\omega \in \mathcal{D}$ and $L \in \mathrm{DDO}_{m}^{n, n}$, then the convolution of $\omega$ and $L, \omega * L$ : $\mathcal{D}^{n, 1} \rightarrow\left(\mathcal{D}^{\prime}\right)^{n, 1}$ is given by the formula

$$
\omega * L=\int \omega\left(e^{i t}\right) L_{t} \frac{d t}{2 \pi}
$$


If $A$ is a DTO, then $\omega * A: \mathcal{D}_{a}^{n, 1} \rightarrow\left(\mathcal{D}_{a}^{\prime}\right)^{n, 1}$ is given by

$$
\omega * A=\int \omega\left(e^{i t}\right) A_{t} \frac{d t}{2 \pi}
$$

Note that if $A=P L \mid \mathcal{D}_{a}^{n, 1}$ and $\omega \in \mathcal{D}$ then an easy consequence of Definition 4.6 is that $\omega * A=P(\omega * L) \mid \mathcal{D}_{a}$. Also observe that if $L=\sum_{\ell} \beta_{\ell} D^{(\ell)} \in \mathrm{DDO}_{m}^{n, n}, \varphi \in \mathcal{D}^{n, 1}$, and $\psi \in \mathcal{D}^{1, n}$, then

$$
(\omega * L)(\varphi)(\psi)=\int \omega\left(e^{i t}\right) L_{t}(\varphi)(\psi) \frac{d t}{2 \pi}
$$

Proposition 4.10. If $L=\sum \beta_{\ell} D^{(\ell)} \in D D O_{m}^{n, n}$ and $\omega \in \mathcal{D}$, then $\omega * L=\sum\left(\omega * \beta_{\ell}\right) D^{(\ell)}$.

Proof. If $\varphi, \psi \in \mathcal{D}^{n, 1}$, then

$$
\begin{aligned}
(\omega * L)(\varphi)\left(\psi^{*}\right) & =\int \omega\left(e^{i t}\right) L_{t}(\varphi)\left(\psi^{*}\right) \frac{d t}{2 \pi} \\
& =\int \omega\left(e^{i t}\right)\left(\sum\left(\beta_{\ell}\right)_{t} D^{(\ell)}\right)(\varphi)\left(\psi^{*}\right) \frac{d t}{2 \pi} \\
& =\sum \int \omega\left(e^{i t}\right)\left(\left(\beta_{\ell}\right)_{t} D^{(\ell)}\right)(\varphi)\left(\psi^{*}\right) \frac{d t}{2 \pi} \\
& =\sum\left(\int \omega\left(e^{i t}\right)\left(\left(\beta_{\ell}\right)_{t} D^{(\ell)}\right) \frac{d t}{2 \pi}\right)(\varphi)\left(\psi^{*}\right) \\
& =\sum\left(\int \omega\left(e^{i t}\right)\left(\beta_{\ell}\right)_{t} \frac{d t}{2 \pi}\right) D^{(\ell)}(\varphi)\left(\psi^{*}\right) \\
& =\left(\sum\left(\omega * \beta_{\ell}\right) D^{(\ell)}\right)(\varphi)\left(\psi^{*}\right)
\end{aligned}
$$

This computation establishes Proposition 4.10.

Proposition 4.11. Fix $\omega \in \mathcal{D}$ with $\omega \geq 0$. If $L$ is a Dirichlet operator, then $\omega * L$ is a Dirichlet operator and $\left\|M_{\omega * L}\right\| \leq\left\|M_{L}\right\|$. If $A$ is an analytic Dirichlet operator, then $\omega * A$ is an analytic Dirichlet operator and $\left\|M_{\omega * A}\right\| \leq\left\|M_{A}\right\|$.

Proof. If ord $L=0$, then $L \geq 0$. Consequently, $\omega * L \geq 0$ and ord $(\omega * L)=0$. Thus $\omega * L$ is a Dirichlet operator if ord $L=0$.

Now suppose ord $L>0$. If $c>1$ and

$$
L-c^{-2} e^{-i \theta} L e^{i \theta} \geq 0,
$$


then

$$
\begin{aligned}
\omega * L-c^{-2} e^{-i \theta}(\omega * L) e^{i \theta} & =\int \omega L_{t} \frac{d t}{2 \pi}-c^{-2} e^{-i \theta} \int \omega L_{t} \frac{d t}{2 \pi} e^{i \theta} \\
& =\int \omega\left(L_{t}-c^{-2} e^{-i \theta} L_{t} e^{i \theta}\right) \frac{d t}{2 \pi} \\
& \geq 0
\end{aligned}
$$

by Proposition 4.2. Hence $\omega * L$ is a Dirichlet operator and $\left\|M_{\omega * L}\right\| \leq\left\|M_{L}\right\|$. The proof of the second assertion of the proposition follows similarly.

Now if $T \in \mathcal{L}(\mathcal{H})$ is an $m$-isometry and $\gamma \in \mathcal{H}^{n, 1}$, then $L=(T, \gamma) \in \mathrm{DDO}_{m-1}^{n, n}$ and $A=P L \mid \mathcal{D}_{a}^{n, 1}$ is an analytic Dirichlet operator. Hence by Proposition 4.11 , if $\omega \geq 0$, then $M_{\omega * L}$ is a $m$-isometry. To identify $M_{\omega * L}$ spatially in terms of $T$ and $\omega$ we introduce the following notations. If $\mu$ is a positive measure and $\mathcal{H}$ is a Hilbert space, we let $L_{\mathcal{H}}^{2}(\mu)$ denote the Hilbert space of $\mathcal{H}$-valued $\mu$-measurable functions $F$ such that

$$
\int\|F(x)\|^{2} d \mu(x)<\infty
$$

If $\mu$ is supported on the circle and $T \in \mathcal{L}(\mathcal{H})$ define $T_{\mu} \in \mathcal{L}\left(L_{\mathcal{H}}^{2}(\mu)\right)$ by setting

$$
\left(T_{\mu} F\right)\left(e^{i t}\right)=e^{i t} T\left(F\left(e^{i t}\right)\right) .
$$

In addition, if $\gamma=\left(\gamma_{r}\right) \in \mathcal{H}^{n, 1}$ define $\gamma_{\mu} \in\left(L_{\mathcal{H}}^{2}(\mu)\right)^{n, 1}$ by requiring

$$
\left(\gamma_{\mu}\right)_{r}\left(e^{i t}\right)=\gamma_{r} \text { for each } r \leq n
$$

We now are able to identify $M_{\omega * L}$.

Proposition 4.12. Let $T \in \mathcal{L}(\mathcal{H})$ be an m-isometry, $\gamma \in \mathcal{H}^{n, 1}$, and $\omega \in \mathcal{D}$ with $\omega \geq 0$. Let $\mu=\omega \frac{d t}{2 \pi} . T_{\mu}$ is an m-isometry and $\left(T_{\mu}, \gamma_{\mu}\right)^{\wedge}=\omega *(T, \gamma) \hat{\text {. }}$

Proof. If $\varphi, \psi \in \mathcal{D}_{a}^{n, 1}$, then

$$
\begin{aligned}
\left(\omega *(T, \gamma)^{\hat{)}}\right)(\varphi)\left(\psi^{*}\right) & =\int((T, \gamma) \hat{)})_{t}(\varphi)\left(\psi^{*}\right) d \mu \\
& =\int(T, \gamma)\left(\varphi_{-t}\right)\left(\psi_{-t}^{*}\right) d \mu \\
& =\int<\varphi_{-t}^{\tau}(T) \gamma, \psi_{-t}^{\tau}(T) \gamma>d \mu \\
& =\int<\varphi^{\tau}\left(e^{i t} T\right) \gamma, \psi^{\tau}\left(e^{i t} T\right) \gamma>d \mu \\
& =\left\langle\varphi^{\tau}\left(T_{\mu}\right) \gamma_{\mu}, \psi^{\tau}\left(T_{\mu}\right) \gamma_{\mu}\right\rangle_{L_{\gamma}^{2}(\mu)} .
\end{aligned}
$$

This computation establishes Proposition 4.12. 
Now, by a matrix measure $\mu$ we shall mean an $n \times n$ matrix $\left(\mu_{r s}\right)$ with entries in the complex Borel measures supported on the unit circle and such that

$$
\left(\mu_{r s}(\triangle)\right) \geq 0
$$

for all Borel sets $\triangle$. If $\mu$ is a matrix measure and $h \in \mathbb{C}^{n}$, then define a scalar measure $\mu_{h, h}$ by setting

$$
\mu_{h, h}(\triangle)=\langle\mu(\triangle) h, h\rangle
$$

Thus, $\mu_{h, h}$ is a positive measure for each $h \in \mathbb{C}^{n}$. Let us agree to say that a matrix measure $\mu$ is weakly definite if $\mu(\partial \mathbb{D})>0$ (i.e. $\mu(\partial \mathbb{D})$ is a strictly positive definite matrix). Observe that trivially if $\mu$ is a matrix measure, $\mu$ is weakly definite if and only if $\mu_{h, h} \neq 0$ for all nonzero $h \in \mathbb{C}^{n}$. Finally, we remark that Lemma 3.5 has the following immediate generalization.

Lemma 4.13. If $L=\sum_{\ell=0}^{m} \beta_{\ell} D^{(\ell)} \in D D O_{m}^{n, n}$ and $L \geq 0$, then $\beta_{m}$ is a matrix measure. If $A=P L \mid \mathcal{D}_{a}^{n, 1} \geq 0$, then $\beta_{m}$ is a matrix measure.

Definition 4.14. If $L=\sum \beta_{\ell} D^{(\ell)} \in \mathrm{DDO}^{n, n}$, then $L$ is smooth if each $\beta_{\ell}$ is a matrix of smooth functions. $L$ is regular if $L$ is smooth and $\beta_{m}>0$ on $\partial \mathbb{D}$ (i.e., the $n \times n$ matrix $\beta_{m}\left(e^{i \theta}\right)$ is strictly positive definite for all $e^{i \theta} \in \partial \mathbb{D}$ where $m=\operatorname{ord}(L)$.

Proposition 4.15. Let $L=\sum_{\ell=0}^{m} \beta_{\ell} D^{(\ell)} \in D D O_{m}^{n, n}$ and let $\omega \in \mathcal{D}$. $\omega * L$ is smooth. If $L \geq 0$ and $\omega \geq 0$, then $\omega * L \geq 0$. If $L \geq 0, \beta_{m}$ is weakly definite, and $\omega>0$, then $\omega * L$ is regular.

Proof. Let $L=\sum \beta_{\ell} D^{(\ell)}$. Since

$$
\omega * L=\sum\left(\omega * \beta_{\ell}\right) D^{(\ell)}
$$

and since $\omega * \beta_{\ell}$, the convolution of a smooth function and a distribution, is smooth, $\omega * L$ is smooth. If $L \geq 0$ and $\omega \geq 0$, then the positivity of $\omega * L$ follows from (4.7).

Now assume that $L \geq 0, \beta_{m}$ is weakly definite, and $\omega>0$. By Proposition 4.10, we need to show that $\omega * \beta_{m}>0$. Using the definition of convolution, and the facts that $\omega>0$ and $\beta_{m}$ is weakly definite we see that

$$
\left\langle\left(\omega * \beta_{m}\right)\left(e^{i t}\right) h, h\right\rangle=\int \omega\left(e^{i(t-\theta)}\right) d\left(\beta_{m}\right)_{h, h}\left(e^{i \theta}\right)>0
$$

Hence $\left(\omega * \beta_{m}\right)\left(e^{i t}\right)>0$ and the proof of Proposition 4.15 is complete. 
We remark before continuing that Proposition 4.10 remains true if the DDO $L$ is replaced by a DTO $A$.

As we have already discussed if one is given an $n$-cyclic $m$-isometry $T$ then there are in general many choices of $n$-cyclic tuple and thus many possible models for $T$ of the form $M_{L}$. Let us agree to say that an $n$-cyclic $m$-isometry $T \in \mathcal{L}(\mathcal{H})$ possesses a Wiener-Hopf form if there exists an $\gamma \in \mathcal{H}^{n, 1}$ such that

$$
(T, \gamma)^{\wedge}=\frac{d \theta}{2 \pi} D^{(m-1)}+\sum_{\ell=0}^{m-2} \beta_{\ell} D^{(\ell)}
$$

and

$$
\gamma \text { is an } n \text {-cyclic tuple for } T \text {. }
$$

Theorem 4.18. If $T \in \mathcal{L}(\mathcal{H})$ is an $n$-cyclic $m$-isometry and if there exists an $n$-cyclic tuple $\gamma$ for $T$ such $(T, \gamma)^{\wedge}$ is regular, then $T$ possesses a Wiener-Hopf form.

Proof. Let

$$
(T, \gamma)^{\hat{N}}=\sum_{\ell=0}^{m-1} \alpha_{\ell} D^{(\ell)}
$$

with $\alpha_{\ell} \in \mathcal{D}^{n, n}$ and $\alpha_{m-1}>0$. By Wiener-Hopf factorization, there exists an outer $G \in \mathcal{D}_{a}^{n, n}$ such that

$$
G\left(e^{i \theta}\right)^{*} G\left(e^{i \theta}\right)=\left(\alpha_{m-1}\left(e^{i \theta}\right)^{\tau}\right)^{-1}
$$

Let $\mu=G(T) \gamma((3.51))$. Since $G^{-1} \in \mathcal{D}_{a}^{n, n}$, Proposition 1.20 and the cyclicity of $\gamma$ implies that $\mu$ is a cyclic $n$-tuple for $T$. Set

$$
(T, \mu)^{\wedge}=\sum_{\ell=0}^{m-1} \beta_{\ell} D^{(\ell)}
$$

To see that $\beta_{m-1}=1$, note that

$$
\begin{aligned}
\beta_{m-1} & =\frac{1}{(m-1) !}\left(\frac{d}{d D}\right)^{m-1}(T, \mu)^{\wedge} \\
& =\frac{1}{(m-1) !} G^{\tau *}\left(\frac{d}{d D}\right)^{m-1}(T, \gamma)^{\wedge} G^{\tau} \\
& =G^{\tau *} \alpha_{m-1} G^{\tau} \\
& =1 .
\end{aligned}
$$


This establishes Theorem 4.18.

\section{References}

[Ag1] Agler, J., Subjordan operators, Thesis, Indiana University, 1980.

[Ag2] Agler, J., "The Arveson extension theorem and coanalytic models," Integral Equations and Operator Theory 5 (1982), 608-631.

[Ag3] Agler, J., An Abstract Approach to Model Theory, Surveys of some recent results in operator theory Vol2, Pitman Research Notes in Mathematics Series; ISSN 0269-3674; 192, 1-24.

[Ag4] Agler, J., "A Disconjugacy Theorem for Toeplitz Operators", American Journal of Mathematics, 112(1990), 1-14.

[Ag5] Agler, J., "Sub-Jordan Operators Bishop's Theorem, Spectral Inclusion and Spectral Sets", J. Operator Theory 7(1982), 373-395.

[Ag6] Agler, J., "Hypercontractions and Subnormality", J. Operator Theory 13(1985), 203217.

[Ag7] Agler, J., " Rational Dilation of an annulus", Annals of Mathematics, 121(1985), 537-563.

[Arv1] Arveson, W.B., "Subalgebras of $C^{*}$-algebras", Acta Math. 123(1969), 141-224.

[Arv2] Arveson, W.B., "Subalgebras of $C^{*}$-algebras II", Acta Math. 128(1972), 271-308.

[B] Beurling, A., "On two problems concerning Linear transformations in Hilbert space," Acta. Math. 81 (1949), 239-255.

[Ber-S] Berger, C.A., Shaw, B.L., "Self-commutators of multicyclic hyponormal operators are always trace class", B.A.M.S., 79(1973), 1193-1199.

[Bir-S] Birman, M. S., and Solomjak, M. Z., Spectral Theory of self-adjoint Operators in Hilbert Space, D. Reidel Publishing Company, Dordrecht/Boston/Lancaster/Tokyo, 1986.

[Br-R] de Branges, L., Rovnyak, J., Square Summable Power Series, New York, Holt, Rinehart and Winston, 1966.

[C] Conway, John B., A Course in Functional Analysis, New York/Berlin/Heidelberg/ Tokyo, Springer-Verlag, 1985.

[Ca] Calkin, J.W., "Two-sided ideals and congruences in the ring of bounded operators in Hilbert space", Ann. of Math. (2) 42(1941), 839-873.

[C-F] Colojoară, I., Foias C., Theory of Generalized spectral Operators, New York, Gordon and Breach, 1968. 
[C-P] Curto, R.E. and Putinar, M., "Existence of non-subnormal polynomially hyponormal operators", B.A.M.S., 25(1991) \#2, 373-378.

[F] Fillmore, P. A., Notes on operator theory, New York, Van Nostrand, 1970.

[Fr] Franks, E., Polynomially subnormal operator tuples, Thesis, University of California, San Diego, 1991.

[Ha] Halmos, P.R., A Hilbert space problem book, Princeton, D. Van Nostrand, 1970.

[H1] Helton, J. W., "Operators with a representation as multiplication by $x$ on a Sobolev space," Colloquia Math. Soc. Janos Bolyai 5, Hilbert Space Operators, Tihany. Hungary (1970), 279-287.

[H2] Helton, J. W., "Infinite dimensional Jordan operators and Sturm-Liouville conjugate point theory," Trans. Amer. Math. Soc. 170 (1972), 305-331.

[H3] Helton, J. W., Operator Theory, analytic functions, matrices, and electrical engineering, Conference Board of the Mathematical Sciences by the American Mathematical Society, 1987.

[Hel] Helson, H., Lectures on Invariant Subspaces, New York, Academic Press, 1964.

[M1] McCullough, S.A., 3-isometries, Thesis, University of California, San Diego, 1987.

[M2] McCullough, S.A., "SubBrownian Operators", J. Operator Theory, 22(1989) 2, 291305 .

[M-G] Boutet de Monvel and V. Guillemin, The spectral theory of Toeplitz operators, Princeton University Press, Princeton N.J., University of Tokyo Press, Tokyo, 1981.

[N] Nielsen, O.A., "Direct Integral Theory," Lecture notes in pure and applied mathematics, vol. 61, Marcel Dekker, Inc, New York and Basel, 1980.

[P] Putnam, C.R., "An inequality for the area of hyponormal spectra", Math.Z., 116(1970), 323-330.

[R.1] Richter, S., "Invariant subspaces of the Dirichlet shift," J. reine agnew. Math. 386 (1988), 205-220.

[R2] Richter, S., "A representation theorem for cyclic analytic two-isometries," Trans. Amer. Math. Soc., to appear.

[Sa] Sarason, D., "Doubly shift-invariant spaces in $H^{2}, " J$. Operator Theory 16 (1986), $75-97$.

[Steg] Stegenga, D.A., "Multipliers of Dirichlet space", Ill. Jour. of Math. 24(1980), 113139.

[St] Stankus, M., Isosymmetric Linear Transformations On Complex Hilbert Space, Thesis, University of California, San Diego, 1993. 
[Sti] Stinespring, W.F., "Positive functions on $C^{*}$-algebras", P.A.M.S. 6(1955), 211-216.

[Sz.-N,F] Sz.-Nagy. Foias, C., Harmonic analysis of operators in Hilbert space, North Holland, Amsterdam, 1970. 